\title{
Mesh requirements for the finite element approximation of problems with sign-changing coefficients
}

\author{
Anne-Sophie Bonnet-Ben Dhia Camille Carvalho Patrick Ciarlet Jr. \\ October 9, 2017, to appear in Numerische Mathematik
}

\begin{abstract}
Transmission problems with sign-changing coefficients occur in electromagnetic theory in the presence of negative materials surrounded by classical materials. For general geometries, establishing Fredholmness of these transmission problems is well-understood thanks to the T-coercivity approach. Moreover, for a plane interface, there exist meshing rules that guarantee an optimal convergence rate for the finite element approximation. We propose here a new treatment at the corners of the interface which allows to design meshing rules for an arbitrary polygonal interface and then recover standard error estimates. This treatment relies on the use of simple geometrical transforms to define the meshes. Numerical results illustrate the importance of this new design.
\end{abstract}

Keywords: T-coercivity, transmission problem, sign-changing coefficient, conforming finite element method, T-conforming mesh.

\section{Introduction and setting of the transmission problem with sign- changing coefficients}

Our aim is to solve the problem

$$
\left\{\begin{array}{l}
\text { Find } u \in H_{0}^{1}(\Omega) \text { such that: } \\
-\operatorname{div}(\sigma \nabla u)=f \text { in } \Omega
\end{array}\right.
$$

where $\Omega \subset \mathbb{R}^{2}$ is a bounded domain partitioned into two regions, $\sigma$ is a scalar, real-valued, sign-changing coefficient, and $f$ is some given data. In electromagnetic theory, this problem can be interpreted as a transmission problem, in a domain composed of a classical dielectric material $(\sigma>0)$, and a negative material $(\sigma<0)$. A negative material can be for example a metal at optical frequencies, or a metamaterial (e.g. $[22,1]$ ), for which some physical parameters become negative (the permittivity for metals, both the permittivity and the permeability for metamaterials). Due to the sign-changing coefficient $\sigma$, well-posedness of problem (1) is not guaranteed. In particular, classical tools such as Lax-Milgram theorem do not apply since the coercivity on $H_{0}^{1}(\Omega) \times H_{0}^{1}(\Omega)$ of the corresponding sesquilinear form

$$
a:(v, w) \mapsto \int_{\Omega} \sigma \nabla v \cdot \overline{\nabla w} d \Omega,
$$

is lost. However, over the past decade, techniques have been developed to establish well-posedness, under appropriate conditions, via the T-coercivity theory: introduced in [5], it consists in building isomorphisms $\mathrm{T}$ such that the form $(v, w) \mapsto a(v, \mathrm{~T} w)$ is coercive on $H_{0}^{1}(\Omega) \times H_{0}^{1}(\Omega)$. For short, we say in this case that $a(\cdot, \cdot)$ is $\mathrm{T}$-coercive. What is less clear is the discrete counterpart of this approach.

In this paper, we consider problem (1) with the following hypothesis on $\sigma$ :

$$
\left\{\begin{array}{l}
\sigma_{\mid \Omega_{1}}=\sigma_{1} \text { is a constant such that } \sigma_{1}>0 \\
\sigma_{\mid \Omega_{2}}=\sigma_{2} \text { is a constant such that } \sigma_{2}<0 .
\end{array}\right.
$$

The ratio $\kappa_{\sigma}:=\sigma_{2} / \sigma_{1}$ is called the contrast. Let $\Omega$ be a domain of $\mathbb{R}^{2}$, that is a connected bounded open subset of $\mathbb{R}^{2}$ with a Lipschitz boundary. It is split as $\bar{\Omega}=\overline{\Omega_{1}} \cup \overline{\Omega_{2}}$, where $\Omega_{1}$ and $\Omega_{2}$ are two disjoint domains. 
The interface separating $\Omega_{1}$ and $\Omega_{2}$ is called $\Sigma$ : we assume that it is a polygonal line made of straight edges and corners. Given $v$ defined over $\Omega$, we use the notation $v_{i}:=v_{\mid \Omega_{i}}, i=1,2$.

The equivalent variational formulation of (1) reads:

$$
\text { Find } u \in V \text { such that } \forall w \in V, a(u, w)=\langle f, w\rangle \text {, }
$$

where $V=H_{0}^{1}(\Omega), a$ is the form defined in $(2)$, and $\langle\cdot, \cdot\rangle$ denotes the duality pairing between $V$ and its dual $V^{\prime}$.

Let us first describe a simple configuration for which everything is well understood. It is the symmetric geometry: $\Sigma$ is a part of a straight line and $\Omega_{2}$ is the symmetric of $\Omega_{1}$ with respect to $\Sigma$. Then one can easily prove that the problem is well-posed if and only if $\kappa_{\sigma} \neq-1$, by considering the following operators $\mathrm{T}$ :

$$
\mathrm{T} v=\left\{\begin{array}{ll}
v_{1} & \text { in } \Omega_{1} \\
-v_{2}+2 \mathrm{~S} v_{1} & \text { in } \Omega_{2}
\end{array}, \text { respectively } \mathrm{T} v=\left\{\begin{array}{ll}
v_{1}-2 \mathrm{~S} v_{2} & \text { in } \Omega_{1} \\
-v_{2} & \text { in } \Omega_{2}
\end{array},\right.\right.
$$

where $\mathbf{S} v_{1}(x)=v_{1}(\mathcal{S} x)$ (resp. $\mathbf{S} v_{2}(x)=v_{2}(\mathcal{S} x)$ ), with $\mathcal{S}$ denoting the symmetry with respect to $\Sigma$. On one hand, one can prove that $a(\cdot, \cdot)$ is T-coercive if $\kappa_{\sigma} \neq-1$. Then problem (3), which is equivalent to the problem

$$
\text { Find } u \in V \text { such that } \forall w \in V, a(u, \mathrm{~T} w)=\langle f, \mathrm{~T} w\rangle \text {, }
$$

is well-posed. On the other hand, if $\kappa_{\sigma}=-1$, the problem (1) is ill-posed since it has a kernel (set of solutions with zero right-hand side $f$ ) which is infinite dimensional. Thus we say that the condition $\kappa_{\sigma} \neq-1$ is optimal for the well-posedness of the continuous problem.

Suppose now that $\kappa_{\sigma} \neq-1$ and that we want to approximate the solution with a conforming finite element method (for short, a cFE method). This leads to the discrete problems

$$
\text { Find } u^{h} \in V^{h} \text { such that } \forall w^{h} \in V^{h}, a\left(u^{h}, w^{h}\right)=\left\langle f, w^{h}\right\rangle,
$$

where $\left(V^{h}\right)_{h}$ denotes a sequence of finite-dimensional subspaces of $V$, with $h$ a positive parameter that goes to 0 . If $\mathrm{T}\left(V^{h}\right) \subset V^{h}$ for small $h$, then T-coercivity can be exploited at the discrete level. In particular, the discrete problem is well-posed and, by a straightforward adaptation of Céa's lemma, the error is controlled by the best approximation error. Hence, we just have to ensure the condition $\mathrm{T}\left(V^{h}\right) \subset V^{h}$, which is achieved in this symmetric geometry by using a symmetric mesh (for a uniform degree of approximation). Let us emphasize that using non-symmetric meshes can deteriorate drastically the convergence of the cFE method when $\kappa_{\sigma}$ is close to -1 (cf. [11]).

The objective of our paper is to generalize this type of result to an arbitrary polygonal interface. This is based on two ingredients:

1) adapting the condition $\mathrm{T}\left(V^{h}\right) \subset V^{h}$ to elementary geometries where $\Sigma$ includes a single corner;

2) for every $h$, relaxing the condition $\mathrm{T}\left(V^{h}\right) \subset V^{h}$ by defining a discrete operator $\mathrm{T}_{h} \in \mathcal{L}\left(V_{h}\right)$ such that $\lim _{h \rightarrow 0}\left\|\mathrm{~T}_{h}-\mathrm{T}\right\|_{\mathcal{L}\left(V_{h} ; V\right)}=0$.

Finally, our aim is to provide meshing rules for an arbitrary geometry, ensuring that the standard convergence rate is recovered, as soon as $\kappa_{\sigma}$ is such that the continuous problem (1) is well-posed. Let us emphasize that in practice, the discrete problem (4) is implemented as it is, the operators $\left(\mathrm{T}_{h}\right)_{h}$ being used only to prove convergence theoretically.

The outline of the paper is as follows. In the next section, we provide a review of the techniques proposed so far to approximate problem (1) with sign-changing coefficients. Section 3 is dedicated to the construction of new explicit T-coercivity operators for elementary geometries whose interface has only one corner. Then in section 4 we develop the theory that allows one to study the well-posedness of problem (1) for an arbitrary polygonal interface, and as a result we derive the optimal condition on the contrast $\kappa_{\sigma}$ to ensure well-posedness. Our aim is to provide tools that can be extended to the discrete problems, namely the approximation of problem (1) by cFE methods. This is the main topic of section 5 , where convergence is derived as soon as the optimal condition on the contrast is fulfilled. Numerical results are presented in section 6 . Finally some concluding remarks are given. 


\section{Historical background: continuous and discrete T-coercivity}

Let us review known results concerning the T-coercivity approach. Introduced in [5], the T-coercivity technique has then been developed in [2]. Similarly to the symmetric case, the idea is to build linear continuous operators T on $V$ :

$$
\mathrm{T} v=\left\{\begin{array}{ll}
v_{1} & \text { in } \Omega_{1} \\
-v_{2}+2 \mathrm{R} v_{1} & \text { in } \Omega_{2}
\end{array}, \text { respectively } \mathrm{T}^{\prime} v=\left\{\begin{array}{ll}
v_{1}-2 \mathrm{R}^{\prime} v_{2} & \text { in } \Omega_{1} \\
-v_{2} & \text { in } \Omega_{2}
\end{array},\right.\right.
$$

where $\mathrm{R}, \mathrm{R}^{\prime}$ are operators (to be precised) such that $\left.\mathrm{R} v_{1}\right|_{\Sigma}=\left.v_{1}\right|_{\Sigma},\left.\mathrm{R}^{\prime} v_{2}\right|_{\Sigma}=\left.v_{2}\right|_{\Sigma}$. This construction is attractive because once the operators $\mathrm{R}, \mathrm{R}^{\prime}$ are settled, the operators $\mathrm{T}, \mathrm{T}^{\prime}$ define isomorphisms of $V$. Let us present the available constructions of $\mathrm{R}$ (note that $\mathrm{R}^{\prime}$ is obtained by inverting the roles of $\Omega_{1}$ and $\Omega_{2}$ ).

The first idea (see [5]) was to consider an operator $\mathrm{R}^{(0)}$ that acts from $H_{00}^{1 / 2}(\Sigma)$ (the space of traces on $\Sigma$ of functions of $V$ ) to $V_{2}$, where $V_{i}:=\left\{v_{i}, v \in V\right\}, i=1,2$. One can choose for instance a harmonic lifting. Then it was proven that $\mathrm{T}$-coercivity is realized with $\mathrm{T}^{(0)}$ (defined as (5) with $\mathrm{R}^{(0)}$ ) under the condition that $\left|\kappa_{\sigma}\right|$ is "small enough" (with no explicit bound). At the discrete level, for $v^{h} \in V^{h}, \mathrm{R}_{h}^{(0)} v_{1}^{h}$ was defined as the discrete-harmonic element of $V_{2}^{h}\left(V_{i}^{h}:=\left\{v_{i}^{h}, v^{h} \in V^{h}\right\}, i=1,2\right)$, with a trace on $\Sigma$ equal to $v_{1 \mid \Sigma}^{h}$.

A generalization was next proposed in $[19,2]$ with an operator $\mathrm{R}$ that acts from $V_{1}$ to $V_{2}$. Let us point out the difference between the two approaches: in $\mathrm{T}^{(0)}, \mathrm{R}^{(0)}$ acts on the trace of $v_{1}$ on $\Sigma$, while in $\mathrm{T}$ (defined as (5) with $\mathrm{R}$ ), $\mathrm{R}$ acts on the whole function $v_{1}$, defined on $\Omega_{1}$. The case of the symmetry-based operator $\mathrm{S}$ (see the introduction) fits into this second category but not in the first one. In that case, T-coercivity is realized under the condition

$$
\kappa_{\sigma} \notin\left[-\left\|\mathrm{R}^{\prime}\right\|^{2} ;-1 /\|\mathrm{R}\|^{2}\right]
$$

$\operatorname{with}\left({ }^{1}\right)$

$$
\|\mathrm{R}\|=\sup _{w_{1} \in V_{1} \backslash\{0\}} \frac{\left\|\nabla \mathrm{R} w_{1}\right\|_{\Omega_{2}}}{\left\|\nabla w_{1}\right\|_{\Omega_{1}}}, \quad \text { and } \quad\left\|\mathrm{R}^{\prime}\right\|=\sup _{w_{2} \in V_{2} \backslash\{0\}} \frac{\left\|\nabla \mathrm{R}^{\prime} w_{2}\right\|_{\Omega_{1}}}{\left\|\nabla w_{2}\right\|_{\Omega_{2}}}
$$

The authors of [19] exhibit a special geometry, different from the symmetric one, where the operators $\mathrm{R}, \mathrm{R}^{\prime}$ can be again built using only axial symmetries.
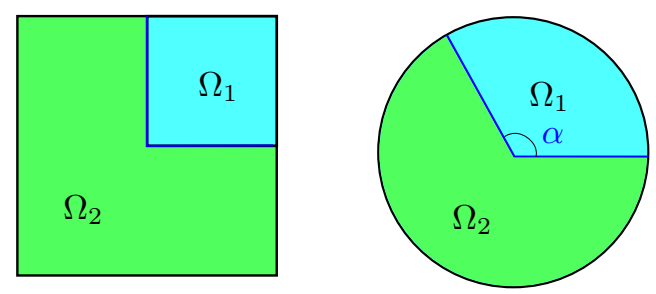

Figure 1: Examples of geometries that have been handled with the T-coercivity: (left) a square where $\Omega_{1}$ is one quadrant; (right) a disk where $\Omega_{1}$ is an angular sector of angle $\alpha$.

This is the case where $\Omega$ is a square, and $\Omega_{1}$ is one quadrant of this square (right-angle geometry, see figure 1-left). One finds $\|\mathrm{R}\|^{2}=\left\|\mathrm{R}^{\prime}\right\|^{2}=3$. On the other hand, the case where $\Omega$ is a disk and $\Omega_{1}$ is an angular sector of angle $\alpha$ is solved in [2] (see figure 1-right). For this second configuration the operators are based on the composition of a central symmetry and an angular dilation. In this case, $\|\mathrm{R}\|^{2}=\left\|\mathrm{R}^{\prime}\right\|^{2}=I_{\alpha}$ where

$$
I_{\alpha}:=\max \left(\frac{2 \pi-\alpha}{\alpha}, \frac{\alpha}{2 \pi-\alpha}\right) .
$$

The result is optimal in the sense that the problem (1)-(3) is ill-posed when $\kappa_{\sigma} \in\left[-I_{\alpha} ;-1 / I_{\alpha}\right]$, cf. [2]. Note that when $\alpha=\pi / 2$, one has $I_{\alpha}=3$, and we remark that the interval in (6) is identical for both geometries.

\footnotetext{
${ }^{1}$ From now on, we denote by $\|\cdot\|_{\mathcal{O}}$ the $L^{2}$-norm over the open set $\mathcal{O}$.
} 
The case of an arbitrary geometry was completely clarified in [2]. It appears that proving the wellposedness of (1)-(3) is too restrictive, and possible only for very simple cases. A more relevant objective is to ensure that the problem is well-posed in the Fredholm sense (see section 4). And it has been proved by a localization process that this property depends only on the value of the contrast $\kappa_{\sigma}$ and on the geometry of the interface $\Sigma$, but not on the global geometry of $\Omega$. The localization is realized with the help of suitably chosen cut-off functions which vanish away from the interface. In particular for a given closed polygonal line $\Sigma$, problem (3) is well-posed in the Fredholm sense if and only if $\kappa_{\sigma} \notin\left[-I_{\alpha} ;-1 / I_{\alpha}\right]$ where $\alpha$ is the smallest corner angle of $\Sigma$ and $I_{\alpha}$ is defined as in (8).

What are the discrete counterparts of these results? The difficulty is that, in general, the imbedding $\mathrm{T}\left(V^{h}\right) \subset V^{h}$ does not hold, due to the presence of the operators $\mathrm{R}, \mathrm{R}^{\prime}$, or due to the presence of the cut-off functions. To address this issue, various approaches have been proposed in [5], [19] and [11]. We refer to the latter for the more complete and detailed numerical analysis. Roughly speaking, in all these previous works, the numerical analysis hinges on the introduction of an approximated operator $\mathrm{T}_{h}$, defined for instance with the help of an interpolation operator. In [5] and [19], the drawback is that convergence of the cFE method is guaranteed under a condition on the contrast which is more restrictive than the one for the continuous problem. An attempt $\left({ }^{2}\right)$ to remove this limitation has been proposed in [11], but only for interfaces without corners. In the following we provide results for an arbitrary polygonal interface. We start with the case where the interface has only one corner.

\section{New T-coercivity operators and associated T-conforming meshes around corners}

Going back to the case of the disk (see figure 1-right), if $\alpha=\pi / 2$ there are at least two alternatives to build the operators $\mathrm{T}, \mathrm{T}^{\prime}$ to prove well-posedness when $\kappa_{\sigma} \notin[-3 ;-1 / 3]$. The first one relies on axial symmetries (similarly to [19]), whereas the second one relies on a composition of a symmetry with respect to the center and an angular dilation [2]. Although these two approaches are equivalent at the continuous level, this is not the case at the discrete level. Indeed, for the first approach it is clear that $\mathrm{T}\left(V_{h}\right) \subset V_{h}$ and $\mathrm{T}^{\prime}\left(V_{h}\right) \subset V_{h}$ if one uses axially symmetric meshes. On the other hand, for the second approach $\mathrm{T}\left(V_{h}\right) \subset V_{h}$ is never satisfied since the polynomial nature of basis FE functions is not preserved by angular dilations. The object of this section is to generalize the first approach to an arbitrary angle. To do so, first we build new operators $\mathrm{R}_{\text {new }}$, $\mathrm{R}_{\text {new }}^{\prime}$ as rotation- and symmetry-based operators such that T-coercivity is realized (using $\mathrm{T}, \mathrm{T}^{\prime}$ defined as (5) with $R_{\text {new }}, R_{\text {new }}^{\prime}$ ) under the same conditions as the case of an angular sector [2]. The key idea is to consider a pattern-based domain $\Omega$ (see figure 2 ).
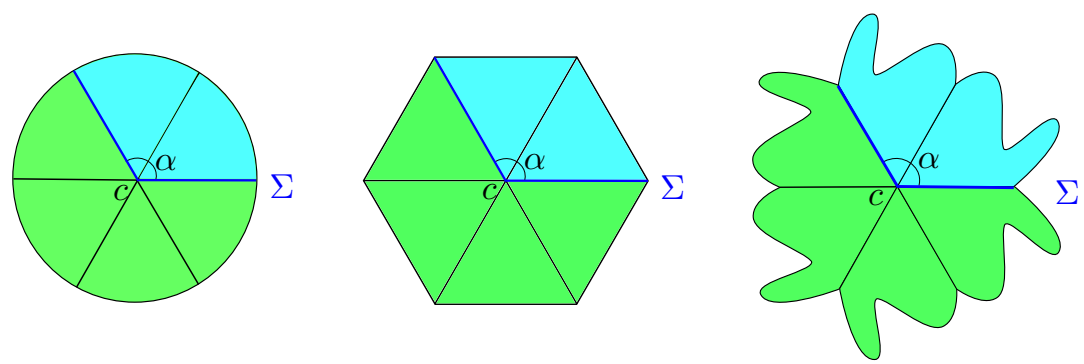

Figure 2: Examples of pattern-based geometries with a corner $c$ of angle $\alpha=2 \pi / 3$ measured in $\Omega_{1}$ : (left) sector-based; (middle) triangle-based; (right) leaf-based geometry.

To define such a domain $\Omega$, let $p, q \in \mathbb{N} \backslash\{0\}$ with $p+q$ even and introduce the angle $\beta:=\frac{2 \pi}{p+q}$, and the cone $C_{\beta}:=\{(\rho \cos \theta, \rho \sin \theta) \mid 0<\rho, 0<\theta<\beta\}$. Finally let us introduce the axial symmetries $\mathcal{S}^{j}$ with respect to

\footnotetext{
${ }^{2}$ It is an attempt only, because there is a mistake in the definition of the discrete operator $\mathrm{T}_{h}$ that will be clarified in section 5 .
} 
the line $\theta=j \beta$ for $j=0, p+q-1$ (the numbering is chosen counterclockwise). Let $\mathscr{P} \subset C_{\beta}$ be a bounded domain that coincides locally at the corner with $C_{\beta}$. A pattern-based domain $\Omega$ is the union of $p+q$ patterns, i.e. $\bar{\Omega}=\bigcup_{j=1}^{p+q} \overline{\mathscr{P}} j$, where $\mathscr{P}_{1}=\mathscr{P}$ and $\mathscr{P}_{j+1}=\mathcal{S}^{j} \mathscr{P}_{j}$ for $j=1, p+q-1$. The condition $p+q$ even ensures that $\mathscr{P}_{1}=\mathcal{S}^{0} \mathscr{P}_{p+q}$. Then $\Omega_{1}$ is composed of the first $p$ patterns, $\overline{\Omega_{1}}=\bigcup_{j=1}^{p} \overline{\mathscr{P}_{j}}$, and $\Omega_{2}$ is composed of the last $q$ patterns, $\overline{\Omega_{2}}=\bigcup_{j=p+1}^{p+q} \overline{\mathscr{P}_{j}}$. In figure 2 , one has $p=2$ and $q=4$ with respectively a sector, a triangle, a leaf as pattern $\mathscr{P}$. Then one has $\alpha:=p \beta=2 \pi \frac{p}{p+q}$ and it follows that

$$
I_{\alpha}:=\max \left(\frac{p}{q}, \frac{q}{p}\right)
$$

Note that for the disk, the condition on the parity of $p+q$ is by no means restrictive. Indeed if $p+q$ is odd, one simply doubles $p$ and $q$ without changing the value of $\alpha$.

For the sake of clarity, we introduce also the following notations:

- $\Omega_{1}^{k}=\mathscr{P}_{k}, k=1, p$, the $k$-th pattern of $\Omega_{1}$.

- $\Omega_{2}^{l}=\mathscr{P}_{p+l}, l=1, q$, the $l$-th pattern of $\Omega_{2}$.

- $v_{1}^{k}:=\left.v\right|_{\Omega_{1}^{k}}, k=1, p$ the restriction to $\Omega_{1}^{k}$. Similarly, $v_{2}^{l}:=\left.v\right|_{\Omega_{2}^{l}}, l=1, q$.

- $e_{n}, n=1,2$, the two edges of $\Sigma$ such that $e_{1}$ coincides locally with $\{(\rho, 0), 0<\rho\}$, while $e_{2}$ coincides locally with the line $\{(\rho \cos \alpha, \rho \sin \alpha), 0<\rho\}$.

To define $\mathrm{R}_{\text {new }}$ as a rotation- and symmetry-based operator from $V_{1}$ to $V_{2}$, one introduces $\mathcal{R}_{m}$ the rotation of angle $m \beta, m=0, p+q-1$. Define also their inverse $\mathcal{R}_{-m}$ the rotation of angle $-m \beta$. These geometrical transforms are such that

- for $(\rho, \theta) \in \Omega_{1}^{k}$, for all $k \in I_{1}:=\llbracket 1, \min (p, q) \rrbracket, \mathcal{S}^{0}(\rho, \theta) \in \Omega_{2}^{q+1-k}$,

- for $(\rho, \theta) \in \Omega_{1}^{k}$, for all $k \in I_{2}:=\llbracket p+1-\min (p, q), p \rrbracket, \mathcal{S}^{p}(\rho, \theta) \in \Omega_{2}^{p+1-k}$,

- for $(\rho, \theta) \in \Omega_{1}^{k}$, for all $k \in \llbracket 1, p \rrbracket, \mathcal{R}_{p+l-k}(\rho, \theta) \in \Omega_{2}^{l}$, for all $l \in \llbracket 1, q \rrbracket$.

Then one defines symmetry-based operators $\mathrm{S}^{n}(n=1,2)$ and rotation-based operators $\mathrm{R}_{m}(m=1, p+q-1)$ from $V_{1}^{k}:=\left\{v_{1}^{k}, v \in V\right\}$ to $V_{2}^{l}:=\left\{v_{2}^{l}, v \in V\right\}$ by:

- $\mathrm{S}^{1} v_{1}^{k}(\rho, \theta)=v_{1}^{k}\left(\mathcal{S}^{0}(\rho, \theta)\right)$ for $(\rho, \theta) \in \Omega_{2}^{q+1-k}$, for all $k \in I_{1}$

- $\mathrm{S}^{2} v_{1}^{k}(\rho, \theta)=v_{1}^{k}\left(\mathcal{S}^{p}(\rho, \theta)\right)$ for $(\rho, \theta) \in \Omega_{2}^{p+1-k}$, for all $k \in I_{2}$,

- $\mathrm{R}_{k-(p+l)} v_{1}^{k}(\rho, \theta)=v_{1}^{k}\left(\mathcal{R}_{k-(p+l)}(\rho, \theta)\right)$ for $(\rho, \theta) \in \Omega_{2}^{l}$, for all $l=1, q$, for all $k=1, p$.

A rotation- and symmetry-based operator is then an ad hoc composition of $\mathrm{S}^{1}, \mathrm{~S}^{2}$ and $\mathrm{R}_{m}$. The general construction of a global, pattern-based, admissible operator $\mathrm{R}$ from $V_{1}$ to $V_{2}$, ensuring $\mathrm{R} v_{\mid \Sigma}=v_{\mid \Sigma}$ is given in the Appendix A.1. We give one illustrative example below with $p<q$, which is equivalent to $\alpha<\pi$ : we build admissible operators $\mathrm{R}$ and $\mathrm{R}^{\prime}$ in this case. We emphasize that the construction of an operator $\mathrm{R}^{\prime}$ in the case $\alpha<\pi$ can be used to build an operator $\mathrm{R}$ in the case $\alpha>\pi$, and vice versa, $\mathrm{cf}$. (5). We consider $\alpha=2 \pi / 3$, with $p=2$ and $q=4$.

Construction of operators R: one can define two admissible operators (illustrated in figure 3 ):

$$
\mathrm{R}_{1}^{\mathrm{adm}} v_{1}=\left\{\begin{array}{ll}
\mathrm{S}^{2} v_{1}^{2} & \text { in } \Omega_{2}^{1} \\
\mathrm{R}_{-2} v_{1}^{2} & \text { in } \Omega_{2}^{2} \\
\mathrm{~S}^{1} v_{1}^{2} & \text { in } \Omega_{2}^{3} \\
\mathrm{~S}^{1} v_{1}^{1} & \text { in } \Omega_{2}^{4}
\end{array} \quad \text { and } \quad \mathrm{R}_{2}^{\mathrm{adm}} v_{1}= \begin{cases}\mathrm{S}^{2} v_{1}^{2} & \text { in } \Omega_{2}^{1} \\
\mathrm{~S}^{2} v_{1}^{1} & \text { in } \Omega_{2}^{2} \\
\mathrm{R}_{-4} v_{1}^{1} & \text { in } \Omega_{2}^{3} \\
\mathrm{~S}^{1} v_{1}^{1} & \text { in } \Omega_{2}^{4}\end{cases}\right.
$$



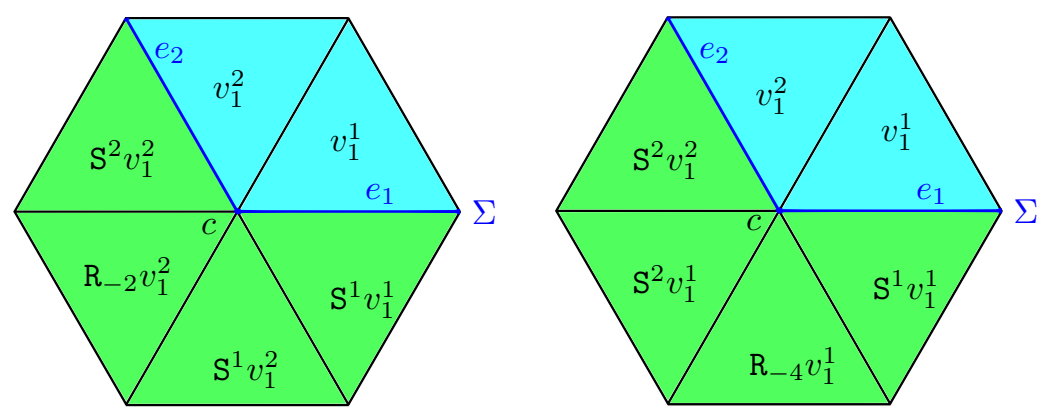

Figure 3: Representation of the two admissible geometry-based operators R.

To realize T-coercivity under the same conditions as in [2], one has to check that these operators $\mathrm{R}$ are optimal in the sense that $\|\mathrm{R}\|^{2}=I_{\alpha}$, where $I_{\alpha}$ is defined in (8) or (9), while the operator norm is defined in (7). For the present example, $I_{\alpha}=2$. Since $\mathrm{S}^{n}$ and $\mathrm{R}_{-m}(n=1,2, m=1,5)$ are isometry-based operators, one finds easily that

$\left\|\nabla\left(\mathrm{R}_{1}^{\mathrm{adm}} v_{1}\right)\right\|_{\Omega_{2}}^{2}=3\left\|\nabla v_{1}^{2}\right\|_{\Omega_{1}^{2}}^{2}+\left\|\nabla v_{1}^{1}\right\|_{\Omega_{1}^{1}}^{2} \leq 3\left\|\nabla v_{1}\right\|_{\Omega_{1}}^{2}$, and $\left\|\nabla\left(\mathrm{R}_{2}^{\mathrm{adm}} v_{1}\right)\right\|_{\Omega_{2}}^{2}=\left\|\nabla v_{1}^{2}\right\|_{\Omega_{1}^{2}}^{2}+3\left\|\nabla v_{1}^{1}\right\|_{\Omega_{1}^{1}}^{2} \leq 3\left\|\nabla v_{1}\right\|_{\Omega_{1}}^{2}$,

with equality for appropriately chosen $v_{1}$, for instance $v_{1}$ such that $v_{1}^{2}=0$ when evaluating $\mathrm{R}_{2}^{\text {adm }}$. According to (6)-(7), this gives $\left\|\mathrm{R}_{1}^{\mathrm{adm}}\right\|^{2}=\left\|\mathrm{R}_{2}^{\mathrm{adm}}\right\|^{2}=3$, which is greater than the expected optimal value of the norm squared. However, considering the average of these two admissible operators gives us the result. More precisely, define

$$
\mathrm{R}_{\text {new }} v_{1}:=\frac{1}{2}\left(\mathrm{R}_{1}^{\text {adm }}+\mathrm{R}_{2}^{\text {adm }}\right) v_{1}=\left\{\begin{array}{ll}
\mathrm{S}^{2} v_{1}^{2} & \text { in } \Omega_{2}^{1} \\
\frac{1}{2}\left(\mathrm{R}_{-2} v_{1}^{2}+\mathrm{S}^{2} v_{1}^{1}\right) & \text { in } \Omega_{2}^{2} \\
\frac{1}{2}\left(\mathrm{~S}^{1} v_{1}^{2}+\mathrm{R}_{-4} v_{1}^{1}\right) & \text { in } \Omega_{2}^{3} \\
\mathrm{~S}^{1} v_{1}^{1} & \text { in } \Omega_{2}^{4}
\end{array},\right.
$$

then one can prove that $\left\|\mathrm{R}_{\text {new }}\right\|^{2}=2$. Indeed, for $v_{1} \in V_{1}$

$$
\left\|\nabla\left(\mathrm{R}_{\mathrm{new}} v_{1}\right)\right\|_{\Omega_{2}}^{2}=\left\|\nabla\left(\mathrm{S}^{2} v_{1}^{2}\right)\right\|_{\Omega_{2}^{1}}^{2}+\left\|\frac{1}{2} \nabla\left(\mathrm{R}_{-2} v_{1}^{2}+\mathrm{S}^{2} v_{1}^{1}\right)\right\|_{\Omega_{2}^{2}}^{2}+\left\|\frac{1}{2} \nabla\left(\mathrm{S}^{1} v_{1}^{2}+\mathrm{R}_{-4} v_{1}^{1}\right)\right\|_{\Omega_{2}^{3}}^{2}+\left\|\nabla\left(\mathrm{S}^{1} v_{1}^{1}\right)\right\|_{\Omega_{2}^{4}}^{2}
$$

and since $\mathrm{S}^{n}$ and $\mathrm{R}_{-m}(n=1,2, m=1,5)$ are isometry-based operators, using the triangle inequality one finds that

$$
\left\|\nabla\left(\mathrm{R}_{\mathrm{new}} v_{1}\right)\right\|_{\Omega_{2}}^{2} \leq\left\|\nabla v_{1}^{2}\right\|_{\Omega_{1}^{2}}^{2}+\left(\frac{1}{2}\left\|\nabla v_{1}^{2}\right\|_{\Omega_{1}^{2}}+\frac{1}{2}\left\|\nabla v_{1}^{1}\right\|_{\Omega_{1}^{1}}\right)^{2}+\left(\frac{1}{2}\left\|\nabla v_{1}^{2}\right\|_{\Omega_{1}^{2}}+\frac{1}{2}\left\|\nabla v_{1}^{1}\right\|_{\Omega_{1}^{1}}\right)^{2}+\left\|\nabla v_{1}^{1}\right\|_{\Omega_{1}^{1}}^{2} .
$$

Define the matrix $M=\left(\begin{array}{cc}0 & 1 \\ 1 / 2 & 1 / 2 \\ 1 / 2 & 1 / 2 \\ 1 & 0\end{array}\right)$, and $\vec{W} \in \mathbb{R}^{2}$ such that $\vec{W}:=\left(\left\|\nabla v_{1}^{1}\right\|_{\Omega_{1}^{1}},\left\|\nabla v_{1}^{2}\right\|_{\Omega_{1}^{2}}\right)^{\top} ;$ by construction, $\|\vec{W}\|_{2}=\left\|\nabla v_{1}\right\|_{\Omega_{1}}$, where $\|\cdot\|_{2}$ denotes the Euclidean norm. Then one has $M \vec{W}=\left(\left\|\nabla v_{1}^{2}\right\|_{\Omega_{1}^{2}}, \frac{1}{2}\left\|\nabla v_{1}^{1}\right\|_{\Omega_{1}^{1}}+\right.$ $\left.\frac{1}{2}\left\|\nabla v_{1}^{2}\right\|_{\Omega_{1}^{2}}, \frac{1}{2}\left\|\nabla v_{1}^{1}\right\|_{\Omega_{1}^{1}}+\frac{1}{2}\left\|\nabla v_{1}^{2}\right\|_{\Omega_{1}^{2}},\left\|\nabla v_{1}^{1}\right\|_{\Omega_{1}^{1}}\right)^{\top}$ and one remarks that

$$
\left\|\nabla\left(\mathrm{R}_{\text {new }} v_{1}\right)\right\|_{\Omega_{2}}^{2} \leq\|M \vec{W}\|_{2}^{2}=\left(M^{\top} M \vec{W}, \vec{W}\right) \leq\left\|M^{\top} M\right\|_{2}\left\|\nabla v_{1}\right\|_{\Omega_{1}}^{2} .
$$

Hence $\left\|\mathrm{R}_{\text {new }}\right\|^{2} \leq\left\|M^{\top} M\right\|_{2}$. A straightforward computation shows that $\left\|M^{\top} M\right\|_{2}=2$. Finally, the equality $\left\|\mathrm{R}_{\text {new }}\right\|^{2}=\left\|M^{\top} M\right\|_{2}$ can be recovered easily $\left({ }^{3}\right)$.

\footnotetext{
${ }^{3}$ For instance, equality is obtained for $\mathrm{R}_{\text {new }}$ by choosing $v_{1}$ such that $v_{1}^{k} \in H_{0}^{1}\left(\Omega_{1}^{k}\right)$, for $k=1, p$, with $v_{1}^{1}$ a symmetric function w.r.t. the line $\theta=\frac{\beta}{2}$, and $v_{1}^{k}(\rho, \theta)=v_{1}^{k}\left(\mathcal{R}_{k-1}(\rho, \theta)\right)$, for $(\rho, \theta) \in \Omega_{1}^{k}$, for $k=2$, $p$. Similarly, equality for $\mathrm{R}^{\prime}$ new is obtained by choosing $v_{2}$ such that $v_{2}^{l} \in H_{0}^{1}\left(\Omega_{2}^{l}\right)$, for $l=1$, $q$, with $v_{2}^{1}$ a symmetric function w.r.t. the line $\theta=\left(p+\frac{1}{2}\right) \beta$, and $v_{2}^{l}(\rho, \theta)=v_{2}^{1}\left(\mathcal{R}_{l-1}(\rho, \theta)\right)$, for $(\rho, \theta) \in \Omega_{2}^{l}$, for $l=2, q$.
} 
Construction of operators $\mathrm{R}^{\prime}$ : in that case one "folds $v_{2}$ up" such that $\mathrm{R}^{\prime} v_{2}$ is defined over $\Omega_{1}$. Then one obtains the two admissible operators described in figure 4 :

$$
\mathrm{R}_{1}^{\prime \text { adm }} v_{2}=\left\{\begin{array}{ll}
\mathrm{S}^{1} v_{2}^{4}-\mathrm{R}_{2} v_{2}^{3}+\mathrm{S}^{2} v_{2}^{2} & \text { in } \Omega_{1}^{1} \\
\mathrm{~S}^{2} v_{2}^{1} & \text { in } \Omega_{1}^{2}
\end{array} \quad, \quad \text { and } \quad \mathrm{R}_{2}^{\mathrm{adm}} v_{2}=\left\{\begin{array}{ll}
\mathrm{S}^{1} v_{2}^{4} & \text { in } \Omega_{1}^{1} \\
\mathrm{~S}^{2} v_{2}^{1}-\mathrm{R}_{4} v_{2}^{2}+\mathrm{S}^{1} v_{2}^{3} & \text { in } \Omega_{1}^{2}
\end{array} .\right.\right.
$$
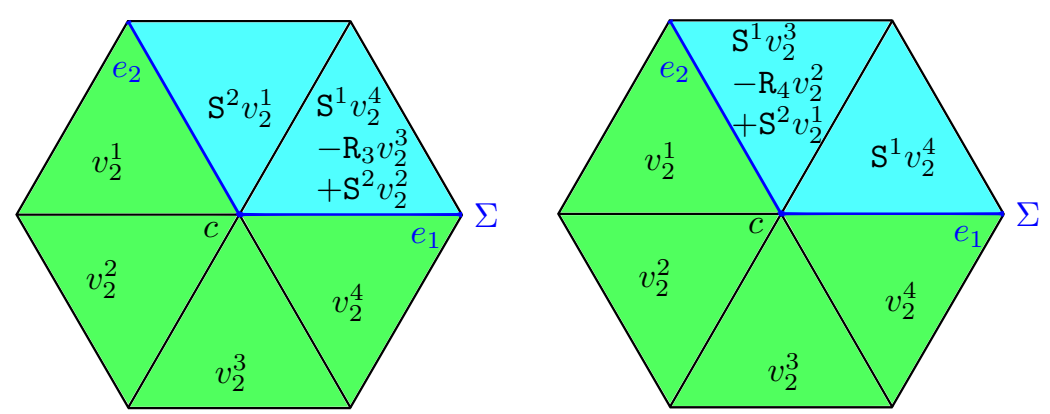

Figure 4: Representation of the two admissible geometry-based operators $\mathrm{R}^{\prime{ }^{\mathrm{adm}}}$ : (left) one applies an axial symmetry in $\Omega_{2}^{1}$ to reach $\Omega_{1}^{2}$ and folds the rest of $v_{2}$ into $\Omega_{1}^{1}$ to ensure continuity; (right) one applies an axial symmetry in $\Omega_{2}^{4}$ to reach $\Omega_{1}^{1}$ and folds the rest of $v_{2}$ into $\Omega_{1}^{2}$ to ensure continuity.

One can operate similarly for the second example by considering $R_{\text {new }}^{\prime} v_{2}$ over $\Omega_{1}$ as the average of the two operators described in figure 4:

$$
\mathrm{R}_{\text {new }}^{\prime} v_{2}=\left\{\begin{array}{ll}
\frac{1}{2}\left(2 \mathrm{~S}^{1} v_{2}^{4}-\mathrm{R}_{2} v_{2}^{3}+\mathrm{S}^{2} v_{2}^{2}\right) & \text { in } \Omega_{1}^{1} \\
\frac{1}{2}\left(2 \mathrm{~S}^{2} v_{2}^{1}-\mathrm{R}_{4} v_{2}^{2}+\mathrm{S}^{1} v_{2}^{3}\right) & \text { in } \Omega_{1}^{2}
\end{array} .\right.
$$

Then one finds $\left\|\mathrm{R}_{\text {new }}^{\prime}\right\|^{2} \leq\left\|M^{\prime} \top M^{\prime}\right\|_{2}=2$, with the matrix $M^{\prime}=\left(\begin{array}{cccc}0 & 1 / 2 & 1 / 2 & 1 \\ 1 & 1 / 2 & 1 / 2 & 0\end{array}\right)=M^{\top}$. Remark that it is expected to find that $M^{\prime}=M^{\top}$ as the role of $p$ and $q$ are exchanged. Thus, one obtains the same results for $\mathrm{R}_{\text {new }}^{\prime}$. We note that equality for the norms, that is $\left\|\mathrm{R}_{\text {new }}^{\prime}\right\|^{2}=\left\|M^{\prime \top} M^{\prime}\right\|_{2}$, can be recovered easily $\left({ }^{3}\right)$. Hence, $\mathrm{R}_{\text {new }}^{\prime}$ is an optimal operator.

Summing up, for any $p, q$, one simply defines $\mathrm{R}_{\text {new }}, \mathrm{R}_{\text {new }}^{\prime}$ as the average of all admissible rotation- and symmetry-based operators. The general expression of these operators is given in the Appendix A.1 and propositions 2 and 3 there give us that for any $p, q, \max \left(\left\|\mathrm{R}_{\text {new }}\right\|^{2},\left\|\mathrm{R}_{\text {new }}^{\prime}\right\|^{2}\right)=I_{\alpha}$.

Let us make some comments regarding this approach:

- this approach applies when the corner angle $\alpha$ can be expressed as a rational fraction times $2 \pi$, that is $\alpha \in 2 \pi \mathbb{Q}$. In the case of an irrational angle $\alpha$, since $\mathbb{Q}$ is dense in $\mathbb{R}$, one can come as close to $\alpha$ as desired (with an increase of the value of $p+q$ ).

- in the case of a general polygonal interface $\Sigma, \Omega$ is locally pattern-based in a neighborhood of any interior corner. Consequently this approach can be adapted using a localization process (see next section).

At this point we have provided optimal operators, that is operators with norm-squared equal to $I_{\alpha}$. Finally we explain how it is possible to satisfy $\mathrm{T}\left(V^{h}\right) \subset V^{h}$ and $\mathrm{T}^{\prime}\left(V^{h}\right) \subset V^{h}$, with $\mathrm{T}, \mathrm{T}^{\prime}$ defined as (5) with these optimal operators. Suppose that $\Omega$ is a pattern-based domain, and that the pattern is polygonal (for instance a triangle). Once this pattern is meshed, the requirement to get T-conforming meshes is to duplicate by symmetry this mesh in each pattern successively. When one considers a uniform degree of approximation on the whole domain, one has automatically $\mathrm{T}\left(V^{h}\right) \subset V^{h}$ and $\mathrm{T}^{\prime}\left(V^{h}\right) \subset V^{h}$ with the new operators described above. Then, as already mentioned, classical errors estimates directly follow from Céa's lemma [11, 
Corollary 1]. Note that there is no need for additional symmetry requirements for the meshing of the pattern.

\section{Numerical illustrations.}

We consider the geometry described previously: $\Omega^{\text {hex }}$ is a hexagon where $\Omega_{1}^{\text {hex }}$ locally coincides with a cone of angle $\alpha=4 \pi / 3$. In this case $I_{\alpha}=2$ and $\left[-I_{\alpha} ;-1 / I_{\alpha}\right]=[-2 ;-1 / 2]$. Let us construct an exact solution (of a problem of type (1) with $\kappa_{\sigma} \notin[-2 ;-1 / 2]$, see (10) below): consider $u_{r} \in H^{1}\left(\Omega^{\text {hex }}\right)$ such that in polar coordinates $\left({ }^{4}\right)$

$$
u_{r}(\rho, \theta)= \begin{cases}\sigma_{1}^{-1} \rho^{2} \sin \left(\frac{p+q}{2}(\theta-\alpha)\right) & \text { in } \Omega_{1}^{\text {hex }} \\ \sigma_{2}^{-1} \rho^{2} \sin \left(\frac{p+q}{2}(\theta-\alpha)\right) & \text { in } \Omega_{2}^{\text {hex }}\end{cases}
$$

where $p$ (resp. $q)$ still denotes the number of patterns in $\Omega_{1}^{\text {hex }}\left(\right.$ resp. $\left.\Omega_{2}^{\text {hex }}\right)$ and $f:=-\operatorname{div}\left(\sigma \nabla u_{r}\right)=\frac{1}{4}((p+$ $\left.q)^{2}-16\right) \sin \left(\frac{p+q}{2}(\theta-\alpha)\right) \in L^{2}\left(\Omega^{\text {hex }}\right)$. By construction, $u_{r}$ is piecewise smooth [17]: $\left.u_{r}\right|_{\Omega_{i}^{\text {hex }}} \in H^{3-\varepsilon}\left(\Omega_{i}^{\text {hex }}\right)$, $\forall \varepsilon>0, i=1,2$. To illustrate the importance of T-conforming meshes around corners, let us add to $u_{r}$ a singular part, that is some $u_{s}(\rho, \theta)=\rho^{\lambda} \Phi(\theta)$, with $\lambda:=\lambda(\sigma) \in \mathbb{R}$, such that $\operatorname{div}\left(\sigma \nabla u_{s}\right)=0$ in $\Omega^{\text {hex }}$. For example we consider $[6,19]$

$$
\begin{aligned}
& \Phi(\theta)=\left\{\begin{array}{l}
\frac{\cosh (\lambda(\theta-\alpha / 2))}{\cosh (\lambda \alpha / 2)} \quad 0 \leq \theta \leq \alpha / 2 \\
\frac{\cosh (\lambda(\theta+(2 \pi-\alpha) / 2))}{\cosh (\lambda(2 \pi-\alpha) / 2)}-(2 \pi-\alpha) / 2 \leq \theta \leq 0
\end{array},\right. \\
& \Phi(\theta)=\Phi(2 \pi-\theta-\alpha / 2) \quad-\pi \leq \theta \leq-(2 \pi-\alpha) \text { or } \alpha / 2 \leq \theta \leq \pi .
\end{aligned}
$$

One chooses for $\lambda$ the smallest possible positive real number. Its value depends on $\kappa_{\sigma}$, and one can prove that since $\kappa_{\sigma} \notin[-2 ;-1 / 2], u_{s} \in H^{1}\left(\Omega^{\text {hex }}\right)[3]$, with $\left.u_{s}\right|_{\Omega_{i}^{\text {hex }}} \in H^{1+\lambda-\varepsilon}\left(\Omega_{i}^{\text {hex }}\right), \forall \varepsilon>0, i=1,2$. Defining $g:=\left.\left(u_{r}+u_{s}\right)\right|_{\partial \Omega^{\mathrm{hex}}}$, one checks that $u=u_{r}+u_{s}$ is the unique solution of the problem

$$
\left\{\begin{array}{l}
\text { Find } u \in H^{1}\left(\Omega^{\text {hex }}\right) \text { such that: } \\
-\operatorname{div}(\sigma \nabla u)=f \quad \text { in } \Omega^{\text {hex }} \\
u=g \quad \text { on } \partial \Omega^{\text {hex }}
\end{array} .\right.
$$

With the help of a lifting of the non-zero boundary condition, one easily checks that problem (10) set in this hexagonal domain is well-posed when $\kappa_{\sigma} \notin[-2 ;-1 / 2]$. We consider two kinds of meshes (see figure 5 ), namely standard meshes (without rotation- or symmetry-based invariance) and T-conforming meshes, and discretize the problem for several FE orders, for two chosen contrasts: $\kappa_{\sigma}=-3$ and $\kappa_{\sigma}=-2.1$ (see figure 6). For this geometry, computations give $\lambda=0.5$ when $\kappa_{\sigma}=-3$, and $\lambda \approx 0.205$ when $\kappa_{\sigma}=-2.1$. Classically the regularity of the solution $u=u_{r}+u_{s}$ is driven by the singular behavior $u_{s}$. Consequently one expects an order of convergence equal to $\lambda$ for the relative errors in $H^{1}$-norm. With figure 6 one concludes that T-conforming meshes ensure optimal convergence speed while, for standard meshes, convergence is more erratic with respect to the mesh size. Note that in figure 6 one does not improve the order of convergence using higher FE orders, due to the low regularity of the solution.

\footnotetext{
${ }^{4}$ With this choice $u_{r}(\cdot, \theta)=0$ for $\theta \in\{n \beta, n \in \mathbb{Z}\}$, then continuity is easily ensured at the crossing of the interfaces.
} 

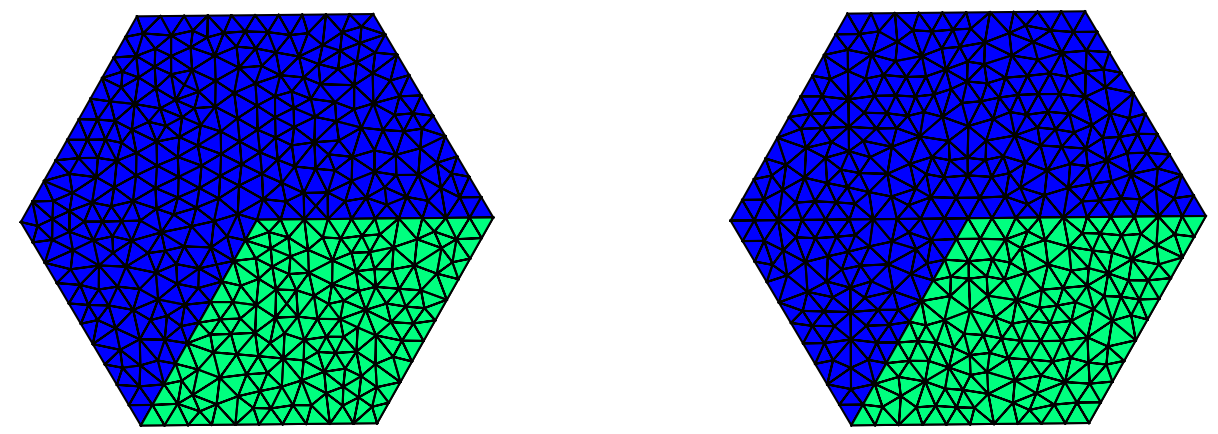

Figure 5: (Left) standard mesh ( $h=0.2,385$ nodes), $\Omega_{1}^{\text {hex }}$ corresponds to blue region while $\Omega_{2}^{\text {hex }}$ corresponds to the green one; (right) T-conforming mesh $(h=0.2,379$ nodes).
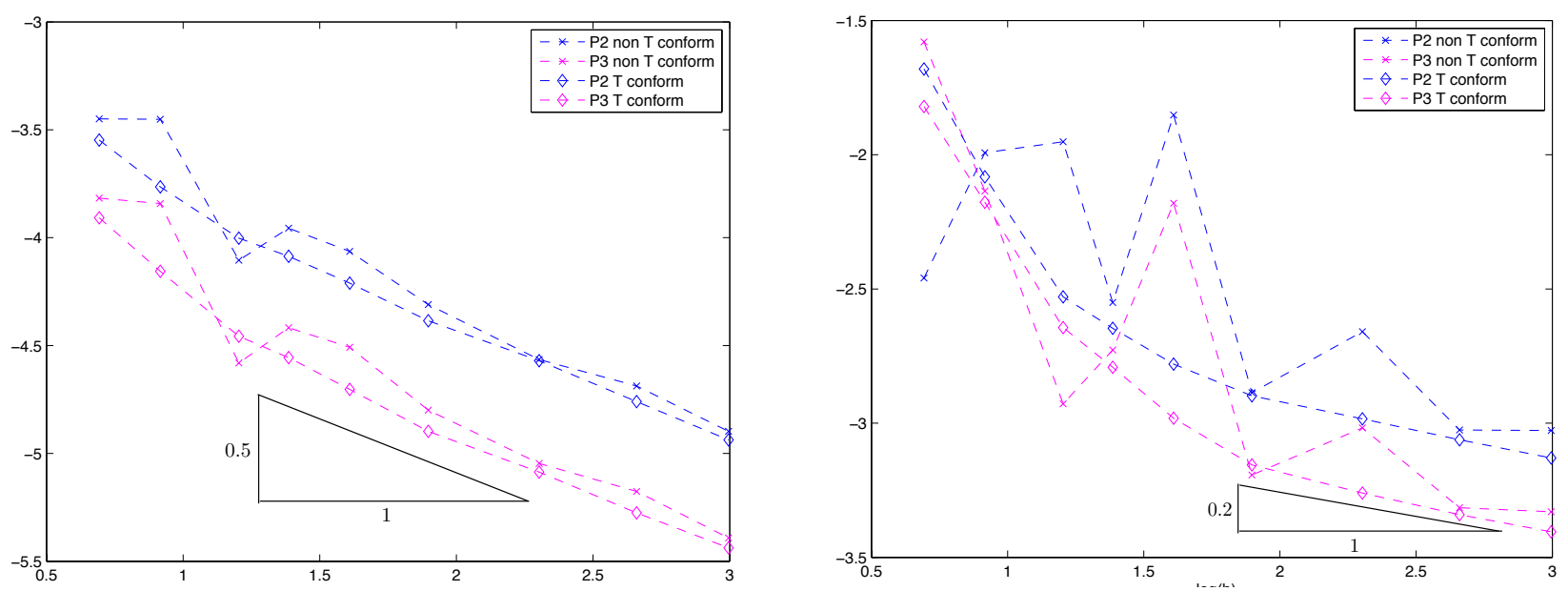

Figure 6: (Left) relative error in $H^{1}$-norm for different mesh sizes $h$ ( $\log$-log scale) for $\kappa_{\sigma}=-3$; (right) relative error in $H^{1}$-norm for different mesh sizes $h$ (log-log scale) for $\kappa_{\sigma}=-2.1$.

In a more general case of a polygonal interface with several interior corners, one has to apply locally this tilings method in the neighborhood of the corners. This strategy is explained in the next two sections.

\section{Weak T-coercivity for a general polygonal interface}

In this section we recall some theoretical results regarding the T-coercivity approach, and prove well-posedness of problem (1) for an arbitrary geometry using the new geometry-based operators introduced in section 3. Consider a Hilbert space $E$ with its dual $E^{\prime}$, a bilinear form $b$ defined over $E \times E$ and $B$ a (linear continuous operator) from $E$ to $E^{\prime}$ such that $\langle B v, w\rangle=b(v, w)$, for all $v, w \in E$. Then, for some data $f \in E^{\prime}$, solving

$$
\text { Find } u \in E \text { such that } b(u, w)=\langle f, w\rangle, \quad \forall w \in E \text {, }
$$

is equivalent to solving

$$
\text { Find } u \in E \text { such that } B u=f \text { in } E^{\prime} \text {. }
$$

Classically $[18,7]$, we recall that $B$ is said to be a Fredholm operator when $\operatorname{dim}(\operatorname{ker}(B))<\infty$, its range $R(B)$ is closed and $\operatorname{codim}(R(B))<\infty$; in this case its index is equal to $\operatorname{dim}(\operatorname{ker}(B))-\operatorname{codim}(R(B))$. If in addition the associated form $b$ is hermitian, the index is automatically equal to 0 . When $B$ is a Fredholm operator of index 0, we say that (11)-(12) is well-posed in the Fredholm sense. On the other hand, (11)-(12) is well-posed 
if, and only if, for all $f \in E^{\prime}$, it has one and only one solution $u$, with continuous dependence: there exists $C>0$ such that, for all $f \in E^{\prime}$, the solution $u$ verifies $\|u\|_{E} \leq C\|f\|_{E^{\prime}}$. In terms of operators, it means that $B^{-1}$ is well-defined as a continuous operator from $E^{\prime}$ to $E$.

To prove well-posedness (in the Fredholm sense) of problem (1), we will apply the theory of T-coercivity $[5,2$, 11]. Let us recall some results. Within the Banach-Necas-Babuska framework, one can define a weak stability condition, also called an inf-sup condition. Below, $\mathcal{L}(E)$ is the set of linear continuous operators defined on $E$, and $\mathcal{K}(E)$ is the subset of compact operators.

Definition 1. Let $b(\cdot, \cdot)$ be a continuous sesquilinear form on $E \times E$.

It verifies $a$ weak stability condition if

$$
\exists \mathrm{C} \in \mathcal{K}(E), \exists \alpha^{\prime}>0, \beta^{\prime} \in \mathbb{R}, \forall v \in E, \sup _{w \in E \backslash\{0\}} \frac{|b(v, w)|}{\|w\|_{E}} \geq \alpha^{\prime}\|v\|_{E}-\beta^{\prime}\|\mathrm{C} v\|_{E} .
$$

Let us now introduce an a priori intermediate condition (cf. [5]).

Definition 2. Let $b(\cdot, \cdot)$ be a continuous sesquilinear form on $E \times E$. It is weakly T-coercive if

$$
\exists \mathrm{C} \in \mathcal{K}(E), \exists \mathrm{T} \in \mathcal{L}(E) \text { bijective, } \exists \underline{\alpha}>0, \underline{\beta} \in \mathbb{R}, \forall v \in E,|b(v, \mathrm{~T} v)| \geq \underline{\alpha}\|v\|_{E}^{2}-\underline{\beta}\|\mathrm{C} v\|_{E}^{2} .
$$

In other words, the form $b(\cdot, \mathrm{T} \cdot)$ fulfills a Gärding's inequality [21].

Remark 1. When $\beta^{\prime} \leq 0$ in (13), one recovers the classical stability condition. Respectively, when $\underline{\beta} \leq 0$ in (14), one obtains T-coercivity for $b(\cdot, \cdot)$.

The operator $\mathrm{T}$ introduced in (14) realizes the inf-sup condition (13): it is sometimes called an inf-sup operator. One can easily prove the following result, see e.g. [8, Chapter 2].

Lemma 1. Let $b(\cdot, \cdot)$ be a continuous, sesquilinear hermitian form on $E \times E$. Then the three assertions below are equivalent:

(i) (11)-(12) is well-posed in the Fredholm sense;

(ii) the form $b$ satisfies a weak stability condition;

(iii) the form $b$ is weakly $\mathrm{T}$-coercive.

As seen in the introduction, problem (1) set in the Hilbert space $V=H_{0}^{1}(\Omega)$ can be expressed in variational form as (3). Let us introduce the linear continuous operator $A$ from $V$ to $V^{\prime}$ such that $\langle A v, w\rangle=a(v, w)$ for all $v, w \in V$, with the form $a$ defined in (2): by construction, $A: v \mapsto-\operatorname{div}(\sigma \nabla v)$. In operator form, (1)-(3) writes equivalently

$$
\text { Find } u \in V \text { such that } A u=f \text { in } V^{\prime} \text {. }
$$

We propose below some realizations of the inf-sup operator that can be used for problem (1) for a polygonal interface $\Sigma$. From now on, we suppose for simplicity that $\Sigma$ is a polygonal line without endpoints, ie. it is a loop. Let $N$ denote the number of its corners, $\left(c_{n}\right)_{n=1, N}$ its corners, $\left(\alpha_{n}\right)_{n=1, N}$ the corner angles measured in $\Omega_{1}$, and $\left(e_{n}\right)_{n=1, N}$ its edges. We introduce the polar coordinates $\left(\rho_{n}, \theta_{n}\right)$ centered at $c_{n}$ such that $\Omega_{1}$ coincides locally with the cone $C_{\alpha_{n}}$.

First, we build a partition of unity on $\bar{\Omega}$. So, let $\left(\chi_{n}\right)_{n=1,2 N} \in\left(C^{\infty}(\bar{\Omega} ;[0,1])\right)^{N}$ with supports localized in a neighborhood of the interface, such that, for $n=1, N, \chi_{n}=1$ near the corner $c_{n}$ and $\chi_{n}=0$ "far" from $c_{n}$ (in particular, $\left(\chi_{n}\right)_{n=1, N}$ have mutually disjoint supports); respectively $\chi_{n+N}=0$ on $\Sigma \backslash e_{n}$. Plus, one imposes the condition $\sum_{n=1,2 N} \chi_{n}=1$ on $\Sigma$. Then we define $\chi_{0}=1-\sum_{n=1,2 N} \chi_{n}$, which vanishes in a neighborhood of the interface $\Sigma$ and, setting $P=2 N$, we obtain that $\left(\chi_{p}\right)_{p=0, P} \in\left(C^{\infty}(\bar{\Omega} ;[0,1])\right)^{P+1}$ is a partition of unity on $\bar{\Omega}$. Finally we denote by $S_{p}:=\operatorname{supp}\left(\chi_{p}\right)$ the support of the cut-off function $\chi_{p}$.

Now, let $\left(\mathrm{R}_{p}\right)_{p=1, P}$ be linear continuous operators that act from $V_{1}$ to $V_{2}$ (resp. $\left(\mathrm{R}_{p}^{\prime}\right)_{p=1, P}$ from $V_{2}$ to $\left.V_{1}\right)$. We suppose in addition that these operators are such that, for all $p=1, P$,

$$
\begin{array}{ll}
\exists C, C^{\prime}>0, \forall w_{1} \in V_{1}, \quad\left\|\mathrm{R}_{p} w_{1}\right\|_{\Omega_{2}} \leq C\left\|w_{1}\right\|_{\Omega_{1}} \quad \text { and } \quad\left\|\chi_{p}^{1 / 2} \nabla\left(\mathrm{R}_{p} w_{1}\right)\right\|_{\Omega_{2} \cap S_{p}} \leq C^{\prime}\left\|\chi_{p}^{1 / 2} \nabla w_{1}\right\|_{\Omega_{1} \cap S_{p}}, \\
\exists C, C^{\prime}>0, \forall w_{2} \in V_{2}, \quad\left\|\mathrm{R}_{p}^{\prime} w_{2}\right\|_{\Omega_{1}} \leq C\left\|w_{2}\right\|_{\Omega_{2}} \quad \text { and } \quad\left\|\chi_{p}^{1 / 2} \nabla\left(\mathrm{R}_{p}^{\prime} w_{2}\right)\right\|_{\Omega_{1} \cap S_{p}} \leq C^{\prime}\left\|\chi_{p}^{1 / 2} \nabla w_{2}\right\|_{\Omega_{2} \cap S_{p}} .
\end{array}
$$


We introduce

$$
\|\mathrm{R}\|:=\max _{p=1, P}\left\|\mathrm{R}_{p}\right\|,\left\|\mathrm{R}^{\prime}\right\|:=\max _{p=1, P}\left\|\mathrm{R}_{p}^{\prime}\right\|, \quad \text { resp. }|\mathrm{R}|:=\max _{p=1, P}\left|\mathrm{R}_{p}\right|,\left|\mathrm{R}^{\prime}\right|:=\max _{p=1, P}\left|\mathrm{R}_{p}^{\prime}\right|,
$$

where for all $p=1, P$,

$$
\begin{gathered}
\left\|\mathrm{R}_{p}\right\|:=\sup _{w_{1} \in V_{1},\left\|\chi_{p}^{1 / 2} \nabla w_{1}\right\|_{\Omega_{1} \cap S_{p}}=1}\left\|\chi_{p}^{1 / 2} \nabla \mathrm{R}_{p} w_{1}\right\|_{\Omega_{2} \cap S_{p}}, \quad\left|\mathrm{R}_{p}\right|:=\sup _{w_{1} \in L^{2}\left(\Omega_{1}\right),\left\|w_{1}\right\|_{\Omega_{1}}=1}\left\|\mathrm{R}_{p} w_{1}\right\|_{\Omega_{2}}, \\
\left\|\mathrm{R}_{p}^{\prime}\right\|:=\sup _{w_{2} \in V_{2},\left\|\chi_{p}^{1 / 2} \nabla w_{2}\right\|_{\Omega_{2} \cap S_{p}}=1}\left\|\chi_{p}^{1 / 2} \nabla \mathrm{R}_{p}^{\prime} w_{2}\right\|_{\Omega_{1} \cap S_{p}}, \quad\left|\mathrm{R}_{p}^{\prime}\right|:=\sup _{w_{2} \in L^{2}\left(\Omega_{2}\right),\left\|w_{2}\right\|_{\Omega_{2}}=1}\left\|\mathrm{R}_{p}^{\prime} w_{2}\right\|_{\Omega_{1}} .
\end{gathered}
$$

Finally, we assume matching conditions on the traces:

$$
\forall p, \forall v_{1} \in V_{1},\left.\mathrm{R}_{p} v_{1}\right|_{\Sigma \cap S_{p}}=\left.v_{1}\right|_{\Sigma \cap S_{p}}, \quad \forall p, \forall v_{2} \in V_{2},\left.\mathrm{R}_{p}^{\prime} v_{2}\right|_{\Sigma \cap S_{p}}=\left.v_{2}\right|_{\Sigma \cap S_{p}} .
$$

Remark 2. At the end of the section, we will show how in practice we can provide $\chi_{p}, \mathrm{R}_{p}, \mathrm{R}_{p}^{\prime}$ which fulfill all the conditions above.

Finally we define the two operators

$$
\mathrm{T} v=\left\{\begin{array}{ll}
v_{1} & \text { on } \Omega_{1} \\
-v_{2}+2 \sum_{p=1, P} \chi_{p} \mathrm{R}_{p} v_{1} & \text { on } \Omega_{2}
\end{array} \quad, \quad \mathrm{~T}^{\prime} v=\left\{\begin{array}{ll}
v_{1}-2 \sum_{p=1, P} \chi_{p} \mathrm{R}_{p}^{\prime} v_{2} & \text { on } \Omega_{1} \\
-v_{2} & \text { on } \Omega_{2}
\end{array} .\right.\right.
$$

Now one can prove the following

Lemma 2. Suppose that $\chi_{p}, \mathrm{R}_{p}, \mathrm{R}_{p}^{\prime}$ satisfy (16) for all $p=1, P$. If the contrast $\kappa_{\sigma}$ does not belong to $\left[-\left\|\mathrm{R}^{\prime}\right\|^{2} ;-1 /\|\mathrm{R}\|^{2}\right]$ where $\|\mathrm{R}\|$ and $\left\|\mathrm{R}^{\prime}\right\|$ are defined by (17)-(18), then the form a is weakly $\mathrm{T}$-coercive for $\mathrm{T}$ or $\mathrm{T}^{\prime}$ defined in (20), and problem (1) is well-posed in the Fredholm sense.

Remark 3. According to [15, 2], the case $\kappa_{\sigma}=-1$ leads to an ill-posed problem (1) in any geometry. Consequently, it follows that $\|\mathrm{R}\| \geq 1,\left\|\mathrm{R}^{\prime}\right\| \geq 1$.

Proof. We assume for instance that $\kappa_{\sigma} \in\left(-1 /\|\mathrm{R}\|^{2} ; 0\right)$. By lemma 1 , we just have to show that the form $a$ is weakly T-coercive, namely

$$
\exists \mathrm{C} \in \mathcal{K}(V), \exists \mathrm{T} \in \mathcal{L}(V), \exists \underline{\alpha}>0, \underline{\beta} \in \mathbb{R}, \forall v \in V, \quad a(v, \mathrm{~T} v) \geq \underline{\alpha}\|v\|_{V}^{2}-\underline{\beta}\|\mathrm{C} v\|_{V}^{2} .
$$

We consider the operator $\mathrm{T}$ defined in (20)-left to prove the above condition. Due to the matching conditions (19) satisfied by $\left(\mathrm{R}_{p}\right)_{p}, \mathrm{~T} v \in V$ for all $v \in V$ and, in addition one checks easily that $\mathrm{T} \circ \mathrm{T}=\mathrm{I}_{V}$ so $\mathrm{T}$ is bijective. Then:

$$
\begin{aligned}
a(v, \mathrm{~T} w) & =b(v, w)+c(v, w), \text { where the forms } b \text { and } c \text { are respectively defined by: } \\
b(v, w) & =\left|\sigma_{1}\right|\left(\nabla v_{1}, \nabla w_{1}\right)_{\Omega_{1}}+\left|\sigma_{2}\right|\left(\nabla v_{2}, \nabla w_{2}\right)_{\Omega_{2}}-2\left|\sigma_{2}\right| \sum_{p=1, P}\left(\nabla v_{2}, \chi_{p} \nabla\left(\mathrm{R}_{p} w_{1}\right)\right)_{\Omega_{2} \cap S_{p}}, \\
c(v, w) & =-2\left|\sigma_{2}\right| \sum_{p=1, P}\left(\nabla v_{2}, \mathrm{R}_{p} w_{1} \nabla \chi_{p}\right)_{\Omega_{2} \cap S_{p}} .
\end{aligned}
$$

First we prove that $b$ is coercive. Using Young's inequality with $\eta>0$ on $b(v, v)$, we get

$$
b(v, v) \geq\left|\sigma_{1}\right|\left\|\nabla v_{1}\right\|_{\Omega_{1}}^{2}+\left|\sigma_{2}\right|\left\|\nabla v_{2}\right\|_{\Omega_{2}}^{2}-\left|\sigma_{2}\right| \sum_{p=1, P}\left(\eta\left\|\chi_{p}^{1 / 2} \nabla v_{2}\right\|_{\Omega_{2} \cap S_{p}}^{2}+\eta^{-1}\left\|\chi_{p}^{1 / 2} \nabla\left(\mathrm{R}_{p} v_{1}\right)\right\|_{\Omega_{2} \cap S_{p}}^{2}\right) .
$$

One has:

$$
\begin{aligned}
\sum_{p=1, P}\left\|\chi_{p}^{1 / 2} \nabla v_{2}\right\|_{\Omega_{2} \cap S_{p}}^{2} & \leq \sum_{p=1, P}\left\|\chi_{p}^{1 / 2} \nabla v_{2}\right\|_{\Omega_{2}}^{2} \\
& =\sum_{p=1, P}\left(\chi_{p} \nabla v_{2}, \nabla v_{2}\right)_{\Omega_{2}} \\
& =\left(\left(1-\chi_{0}\right) \nabla v_{2}, \nabla v_{2}\right)_{\Omega_{2}} \leq\left\|\nabla v_{2}\right\|_{\Omega_{2}}^{2} \text {, as }\left\|1-\chi_{0}\right\|_{L^{\infty}\left(\Omega_{2}\right)}=1 .
\end{aligned}
$$


Next, using the definitions of $\left(\left\|\mathrm{R}_{p}\right\|\right)_{p=1, P}$ and $\|\mathrm{R}\|$, we note that:

$$
\begin{aligned}
\sum_{p=1, P}\left\|\chi_{p}^{1 / 2} \nabla\left(\mathrm{R}_{p} v_{1}\right)\right\|_{\Omega_{2} \cap S_{p}}^{2} & \leq \sum_{p=1, P}\left\|\mathrm{R}_{p}\right\|^{2}\left\|\chi_{p}^{1 / 2} \nabla v_{1}\right\|_{\Omega_{1} \cap S_{p}}^{2} \\
& \leq\|\mathrm{R}\|^{2} \sum_{p=1, P}\left\|\chi_{p}^{1 / 2} \nabla v_{1}\right\|_{\Omega_{1}}^{2} \\
& =\|\mathrm{R}\|^{2}\left(\left(1-\chi_{0}\right) \nabla v_{1}, \nabla v_{1}\right)_{\Omega_{1}} \leq\|\mathrm{R}\|^{2}\left\|\nabla v_{1}\right\|_{\Omega_{1}}^{2} .
\end{aligned}
$$

Hence,

$$
b(v, v) \geq\left(\left|\sigma_{1}\right|-\left|\sigma_{2}\right| \eta^{-1}\|\mathrm{R}\|^{2}\right)\left\|\nabla v_{1}\right\|_{\Omega_{1}}^{2}+\left|\sigma_{2}\right|(1-\eta)\left\|\nabla v_{2}\right\|_{\Omega_{2}}^{2} .
$$

Because $\kappa_{\sigma} \in\left(-1 /\|\mathrm{R}\|^{2} ; 0\right)$, we can choose $\eta$ such that $\|\mathrm{R}\|^{2}\left|\kappa_{\sigma}\right|<\eta<1$, which is equivalent to

$$
\left|\sigma_{1}\right|-\left|\sigma_{2}\right| \eta^{-1}\|\mathrm{R}\|^{2}>0 \quad \text { and } \quad\left|\sigma_{2}\right|(1-\eta)>0 .
$$

Regarding $c(v, v)$, if we let $\mathrm{C}: V \rightarrow V$ such that $(\mathrm{C} v, w)_{V}=c(v, w)$ for all $v, w \in V$, then by Cauchy-Schwarz inequality one gets

$$
\|\mathrm{C} v\|_{V}^{2}=c(v, \mathrm{C} v) \leq G\|\nabla v\|_{\Omega}\|\mathrm{C} v\|_{\Omega}
$$

where $G:=2 P|\mathrm{R}|\left|\sigma_{2}\right| \max _{p=1, P}\left(\left|\chi_{p}\right|_{W^{1, \infty}\left(\Omega_{2}\right)}\right)$. By Rellich's theorem, one concludes that $\mathrm{C}$ is compact. Then by using Young's inequality with $\eta^{\prime}>0$ we find

$$
c(v, v) \geq-\frac{1}{2}\left(\left(\eta^{\prime}\right)^{-1}\|\mathrm{C} v\|_{V}^{2}+\eta^{\prime}\|v\|_{V}^{2}\right),
$$

which ends the proof in the case $\kappa_{\sigma} \in\left(-1 /\|\mathrm{R}\|^{2} ; 0\right)$, by choosing $\eta^{\prime}$ small enough.

On the other hand, if $\kappa_{\sigma} \in\left(-\infty ;-\left\|\mathrm{R}^{\prime}\right\|^{2}\right)$, one can reverse the roles of $\Omega_{1}$ and $\Omega_{2}$ by using this time the operator $\mathrm{T}^{\prime}$ defined in (20)-right. The proof then proceeds as above to prove that there exist $\underline{\alpha}^{\prime}>0, \beta^{\prime}$, and a compact operator $\mathrm{C}^{\prime}$ such that

$$
\forall v \in V, \quad a\left(v, \mathrm{~T}^{\prime} v\right) \geq \underline{\alpha}^{\prime}\|v\|_{V}^{2}-\underline{\beta}^{\prime}\left\|\mathbf{C}^{\prime} v\right\|_{V}^{2},
$$

which is the condition (14).

To conclude the study of the well-posedness of problem (1), let us explicit $\chi_{p},\left(\mathrm{R}_{p}\right)_{p=1, P}$, (resp. $\left.\left(\mathrm{R}_{p}^{\prime}\right)_{p=1, P}\right)$, and compute the bounds. In the case where $\Sigma$ is a polygonal line with all angles $\alpha_{n} \in 2 \pi \mathbb{Q}, n=1, N$, one can explicit these bounds using the results of section 3. To do so, let us define $\left(B_{p}\right)_{p=1, P}$ a sequence of connected open sets so that $\bigcup_{p=1, P} B_{p}$ is a neighborhood of the interface. For all $n=1, N$, define $B_{n}$ as a triangle-based neighborhood of $c_{n}$, and $B_{N+n}$ as a neighborhood of a part of $e_{n}$ (excluding its endpoints) which is symmetric with respect to $e_{n}$ (see figure 7). More precisely, for all $n=1, N, B_{n}$ is a cyclic polygon centered at $c_{n}$ composed of $p_{n}>0$ triangles in $\Omega_{1}$ and $q_{n}>0$ triangles in $\Omega_{2}$, with $p_{n}+q_{n}$ even: define $\rho_{c_{n}}$ the radius and $s_{n}$ the side length of this polygon. Then for all $n=1, N$ one defines $B_{N+n}$ as a trapezoid-based open set with one trapezoid in $\Omega_{1}$ and one in $\Omega_{2}$, each trapezoid being of side lengths $s_{n}$ and $s_{n+1}\left({ }^{5}\right)$. For technical purposes one chooses $p_{n} \geq 2$ so that all part edge neighborhoods $B_{N+n}, n=1, N$ are mutually disjoint open sets. Note that a part edge neighborhood intersects with two corners neighborhoods. Namely for $n=1, N-1$, one has $\bar{B}_{N+n} \cap \bar{B}_{n} \neq \emptyset, \bar{B}_{N+n} \cap \bar{B}_{n+1} \neq \emptyset$, and for $n=N$ one has $\bar{B}_{P} \cap \bar{B}_{N} \neq \emptyset$, $\bar{B}_{P} \cap \bar{B}_{1} \neq \emptyset$. Then one defines $\left(\mathrm{R}_{p}\right)_{p=1, P}$ from $V_{1}$ to $V_{2}$ (and also from $L^{2}\left(\Omega_{1}\right)$ to $L^{2}\left(\Omega_{2}\right)$ ) such that for all $w_{1} \in V_{1}$ and for all $n=1, N$

$$
\mathrm{R}_{n} w_{1}(x)=\left\{\begin{array}{ll}
0 & \text { if } x \notin B_{n} \\
\mathrm{R}_{\text {new }}^{n} w_{1}(x) & \text { if } x \in B_{n}
\end{array},\right.
$$

with $\mathrm{R}_{\text {new }}^{n}$ defined as in section 3 (the general expression is given in Appendix A.1), and

$$
\mathrm{R}_{N+n} w_{1}(x)=\left\{\begin{array}{ll}
0 & \text { if } x \notin B_{N+n} \\
w_{1}\left(x_{\Sigma},-y_{\Sigma}\right) & \text { if } x \in B_{N+n}
\end{array},\right.
$$

\footnotetext{
${ }^{5} B_{2 N}$ is composed of two trapezoids of side lengths $s_{2 N}$ and $s_{1}$.
} 
where $\left(x_{\Sigma}, y_{\Sigma}\right)$ denotes the local cartesian coordinates, and the first axis coincides with $\Sigma$.

Finally, let us precise some properties the cut-off functions $\left(\chi_{p}\right)_{p}$. Recall that $\left(\rho_{p}, \theta_{p}\right)$ denote the polar coordinates centered at $c_{p}, p=1, N$. For all $p=1, N, \chi_{p}(x)=\chi_{p}\left(\rho_{p}\right)$, and $\chi_{p}=1$ for $0 \leq \rho_{p} \leq \rho_{\text {min }, p}$ for some $\rho_{\min , p}>0$; respectively $\chi_{N+p}$ is a symmetric function with respect to the interface. This can be realized by introducing $\psi \in C^{\infty}(\bar{\Omega} ;[0,1])$ equal to 1 in a neighborhood of the interface, for which we remark that $\psi-\sum_{p=1, N} \chi_{p}$ is a (smooth) function that vanishes in a neighborhood of the corners, and also that "close" to the interface, it is a function which is symmetric with respect to the interface $\left({ }^{6}\right)$. Then one chooses $S_{p}$ the support of $\chi_{p}$ such that $S_{p} \subset B_{p}$ for the corner case $(p=1, N)\left({ }^{7}\right)$ and in the edge case $S_{p} \subset \overline{B_{p}} \cup \overline{B_{p-N}} \cup \overline{B_{p+1-N}}(p=N+1, P-1)$, resp. $S_{P} \subset \overline{B_{P}} \cup \overline{B_{N}} \cup \overline{B_{1}}$ (see figure 7). One can check that (19) is satisfied for all $\mathrm{R}_{p}, p=1, P$. We can finally state the result on the well-posedness of problem (1).

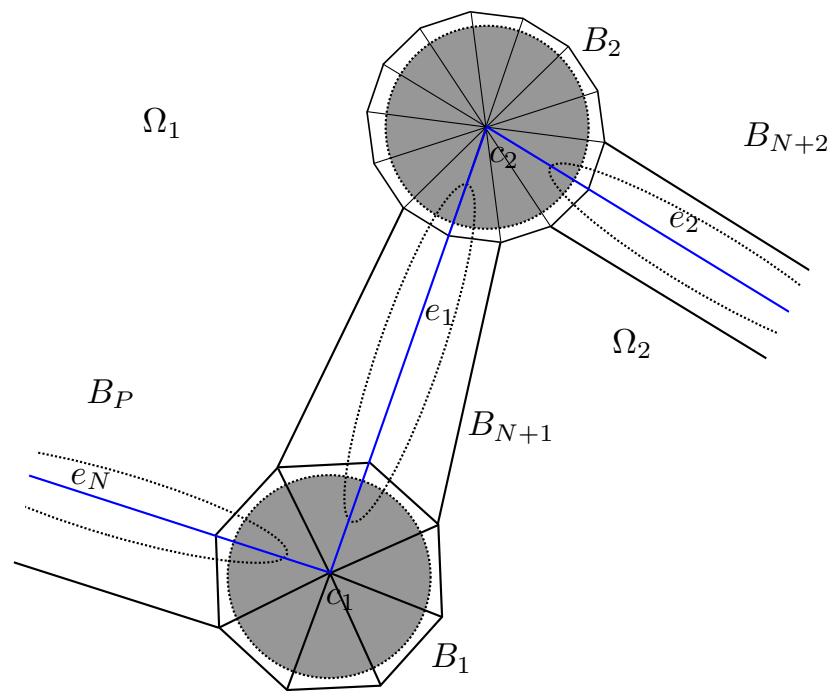

Figure 7: Notations around two corners $c_{1}$ and $c_{2}$ : here $p_{1}=2, q_{1}=6, p_{2}=11$ and $q_{2}=3$. The gray zones represent the support of $\chi_{p}$ while the empty-dashed domains represent the support of $\chi_{N+p}, p=1, N$.

Theorem 1. Assume that all the corners' angles of the interface belong to $2 \pi \mathbb{Q}$. If $\kappa_{\sigma} \notin\left[-I_{\alpha} ;-1 / I_{\alpha}\right]$, with $I_{\alpha}:=\max _{n=1, N} \max \left(\frac{2 \pi-\alpha_{n}}{\alpha_{n}}, \frac{\alpha_{n}}{2 \pi-\alpha_{n}}\right)$, then the form a is weakly T-coercive and problem (1) is well-posed in the Fredholm sense.

Proof. With the operators (21)-(22) and using propositions 4 and 5 (cf. Appendix A.1), one finds that $\min \left(\|\mathrm{R}\|^{2},\left\|\mathrm{R}^{\prime}\right\|^{2}\right) \geq 1$ and $\max \left(\|\mathrm{R}\|^{2},\left\|\mathrm{R}^{\prime}\right\|^{2}\right) \leq I_{\alpha}$. Consequently $\left[-\left\|\mathrm{R}^{\prime}\right\|^{2} ;-1 /\|\mathrm{R}\|^{2}\right] \subseteq\left[-I_{\alpha} ;-1 / I_{\alpha}\right]$. Then if $\kappa_{\sigma} \notin\left[-I_{\alpha} ;-1 / I_{\alpha}\right]$ the result follows from lemma 2 .

Note that if $\kappa_{\sigma} \in\left[-I_{\alpha} ;-1 / I_{\alpha}\right]$, then the problem (1) is not well-posed in the Fredhom sense, according to section 6 of [2]. Hence, we conclude that the condition on the contrast $\kappa_{\sigma} \notin\left[-I_{\alpha} ;-1 / I_{\alpha}\right]$ is optimal for a $2 \mathrm{D}$ geometry with a polygonal interface separating the two subdomains. We define then the critical interval $I_{c}:=\left[-I_{\alpha} ;-1 / I_{\alpha}\right]$.

\section{T-conforming meshes for a general polygonal interface and error estimates}

We study the approximation of problem (1) when the contrast $\kappa_{\sigma}$ does not belong to the critical interval $\left[-I_{\alpha} ;-1 / I_{\alpha}\right]$. According to the previous section (see theorem 1), it follows that problem (1) is well-posed in the Fredholm sense (namely there might be a finite dimensional kernel). From now on, we impose further

\footnotetext{
${ }^{6}$ The strictly positive, upper limit distance up to which this property applies is a function of $\min _{p} \rho_{\min , p}$ and of $\left(\alpha_{p}\right)_{p=1, N}$.

${ }^{7}$ In accordance with the previous notations, one chooses $\rho_{\min , p}$ such that $\rho_{\min , p}<\rho_{c_{p}}$.
} 
that it is well-posed, to ensure the uniqueness of the solution $u$ to be approximated. We study the family of discrete problems (4), focusing on conforming $\left({ }^{8}\right)$ finite element approximations. Our aim is to prove that they can be solved in geometries with interfaces that include corners, and to prove optimality of the approximation, that is the unique solution $u^{h}$ tends towards the exact solution $u$ when $h$ tends to 0 with optimal convergence rate. The geometry and notations are those of section 4 .

To fix ideas, we assume throughout section 5 that $\kappa_{\sigma} \in\left(-1 / I_{\alpha} ; 0\right)$ so that we can use the operator $\mathrm{T}$ as defined in (20)-left. The conforming approximations in $V$ are defined on meshes that match with the interface (all elements are subsets of either $\overline{\Omega_{1}}$ or $\overline{\Omega_{2}}$ ), with piecewise polynomial approximations. Let us consider $\left(\mathcal{T}_{h}\right)_{h}$ a regular family of meshes of $\bar{\Omega}$, made of triangles; for all triangles $\tau$, one has either $\tau \subset \overline{\Omega_{1}}$ or $\tau \subset \overline{\Omega_{2}}$. Define the family of Lagrange FE spaces (for a degree of approximation $d \geq 1$ )

$$
V_{(d)}^{h}:=\left\{v \in V: v_{\mid \tau} \in \mathbb{P}_{d}(\tau), \forall \tau \in \mathcal{T}_{h}\right\}
$$

where $\mathbb{P}_{d}(\tau)$ is the space of polynomials of degree at most $d$ on the triangle $\tau$. Unless otherwise specified, the index $_{(d)}$ is omitted. The parameter, or meshsize, $h$ is defined as $h:=\max _{\tau \in \mathcal{T}_{h}} h_{\tau}$, where $h_{\tau}$ is the diameter of $\tau$. We recall that $V_{1}^{h}$ (resp. $V_{2}^{h}$ ) denotes the FE subspace of $V_{1}$ (resp. $V_{2}$ ) built on the triangulation $\mathcal{T}_{h}$. Throughout this section, $C$ is used to denote a generic positive constant which is independent of the meshsize, the triangulation, and the data/unknown of interest.

Definition 3. Given $h$, let $\mathcal{T}_{h, i}^{p}:=\left\{\tau \in \mathcal{T}_{h}: \tau \cap \operatorname{int}\left(S_{p}\right) \cap \Omega_{i} \neq \emptyset\right\}$, for $i=1,2$ and $p=1, P$.

The meshes $\left(\mathcal{T}_{h}\right)_{h}$ are locally T-conform if, for all $h$ small enough, for all $p=1, P$, for all $\tau \in \mathcal{T}_{h, 1}^{p}$, the image of $\tau$ by the geometrical transforms underlying $\mathrm{R}_{p}$ belongs to $\mathcal{T}_{h, 2}^{p}$.

In practice, the above definition simply imposes that the meshes are locally: invariant by the axial symmetry for each part edge; invariant by the axial symmetries $\mathcal{S}^{j}$ from one pattern, $\mathcal{P}_{j}$, to the next, $\mathcal{P}_{j+1}$, for $j=1, p+q-1$, at each corner. Note that if the conditions of the above definition are met, then for all $v^{h} \in V^{h}$, it holds that $\mathrm{R}_{p} v_{1}^{h}$ is equal to the restriction of some element of $V_{2}^{h}$ on $\Omega_{2} \cap S_{p}$. We set $\Omega_{2}^{h}:=\operatorname{int}\left(\bigcup_{p=1, P} \cup_{\tau \in \mathcal{T}_{h, 2}^{p}} \tau\right)$.

As mentioned in section 2, in general, for $v^{h} \in V^{h}, \mathrm{~T} v^{h} \notin V^{h}$ due to the cut-off functions $\left(\chi_{p}\right)_{p=1, P}$ that need to be interpolated. In other words, the condition $\mathrm{T}\left(V^{h}\right) \subset V^{h}$ is false in general. However one can look for a relaxed condition guaranteeing optimality of the approximation by introducing a discrete counterpart $\mathrm{T}_{h}$ of $\mathrm{T}$ such that $\mathrm{T}_{h}\left(V^{h}\right) \subset V^{h}$ and $\lim _{h \rightarrow 0}\left\|\mathrm{~T}_{h}-\mathrm{T}\right\|_{\mathcal{L}\left(V_{h}\right)}=0$. Our goal in this section is to detail these steps, using some discrete operators $\left(\mathrm{T}_{h}\right)_{h}$ (precised below).

In [11] it was proposed to replace $\chi_{p}$ by its interpolation of degree 1 denoted by $\chi_{p, h}$. To fit with the definition of $\mathrm{T}$ in (20)-left, it leads to consider:

$$
\mathrm{T}_{h}^{0} v^{h}=\left\{\begin{array}{ll}
v_{1}^{h} & \text { in } \Omega_{1} \\
-v_{2}^{h}+2 \sum_{p=1, P} \chi_{p, h} \mathrm{R}_{p} v_{1}^{h} & \text { in } \Omega_{2}
\end{array} .\right.
$$

Unfortunately, for all $p=1, P$, the degree of $\chi_{p, h} \mathrm{R}_{p} v_{1}^{h}$ (restricted to a triangle) is too high. For instance, if one is dealing with Lagrange FE of degree $1(d=1)$, its degree is 2 : hence, $\mathrm{T}_{h}^{0} v^{h}$ does not belong to $V^{h}$ in general. This is why we introduce instead the discrete operator:

$$
\mathrm{T}_{h} v^{h}=\left\{\begin{array}{ll}
v_{1}^{h} & \text { in } \Omega_{1} \\
-v_{2}^{h}+2 I_{h}\left(\sum_{p=1, P} \chi_{p, h} \mathrm{R}_{p} v_{1}^{h}\right) & \text { in } \Omega_{2}
\end{array},\right.
$$

with $I_{h}$ the Lagrange-interpolation operator on $V_{(d)}^{h}$. Moreover from [16, Corollaries 1.109-1.110] we have for $p=1, P$, the stability and approximation estimates

$$
\exists C>0,\left\|\chi_{p, h}\right\|_{W^{1, \infty}\left(\Omega_{2}\right)} \leq C\left\|\chi_{p}\right\|_{W^{1, \infty}\left(\Omega_{2}\right)},
$$

$$
\exists C>0, \forall h \text { small enough, }\left\|\chi_{p}-\chi_{p, h}\right\|_{W^{1, \infty}\left(\Omega_{2}\right)} \leq C h\left|\chi_{p}\right|_{W^{2, \infty}\left(\Omega_{2}\right)} .
$$

\footnotetext{
${ }^{8} \mathrm{~A}$ Discontinuous Galerkin approach has been studied in [12].
} 
We emphasize that the analysis presented in [11] remains valid if one replaces $\mathrm{T}_{h}^{0}$ by $\mathrm{T}_{h}$, as we explain below. Our aim is to prove that the form $a$ is uniformly weakly $\mathrm{T}$-coercive, namely

$$
\exists \underline{\alpha}^{\prime}>0, \underline{\beta} \in \mathbb{R}, \forall h \text { small enough, } \forall v^{h} \in V^{h},\left|a\left(v^{h}, \mathrm{~T}_{h} v^{h}\right)\right| \geq \underline{\alpha}^{\prime}\left\|v^{h}\right\|_{V}^{2}-\underline{\beta}\left\|\mathrm{C} v^{h}\right\|_{V}^{2},
$$

and from there one can follow the last part of the proof of Proposition 3 in [11] to derive a uniform discrete inf-sup condition (by contradiction). Classically, this yields an error estimate.

In order to obtain (27), we shall evaluate $\left|a\left(v^{h},\left(\mathrm{~T}-\mathrm{T}_{h}\right) v^{h}\right)\right|$ for all $v^{h} \in V^{h}$. More precisely we want to prove

$$
\exists C_{0}>0, \forall h \text { small enough, } \forall v^{h} \in V^{h},\left|a\left(v^{h},\left(\mathrm{~T}-\mathrm{T}_{h}\right) v^{h}\right)\right| \leq C_{0} h\left\|v^{h}\right\|_{V}^{2},
$$

which is a consequence of proving that there exists $C>0$ such that, for all $h$ small enough, for all $v^{h} \in V^{h}$, $\left\|\left(\mathrm{T}-\mathrm{T}_{h}\right) v^{h}\right\|_{V} \leq C h\left\|v^{h}\right\|_{V}$. This result is the object of lemma 3 .

Lemma 3. Assume that the meshes are locally T-conform then there holds

$$
\exists C>0, \forall h \text { small enough, } \forall v^{h} \in V^{h},\left\|\left(\mathrm{~T}-\mathrm{T}_{h}\right) v^{h}\right\|_{V} \leq C h\left\|v^{h}\right\|_{V},
$$

so the form a is uniformly weakly $\mathrm{T}$-coercive.

Proof. Given $v^{h} \in V^{h}$, let us apply the triangle inequality:

$$
\left\|\left(\mathrm{T}-\mathrm{T}_{h}\right) v^{h}\right\|_{V} \leq\left\|\left(\mathrm{T}-\mathrm{T}_{h}^{0}\right) v^{h}\right\|_{V}+\left\|\left(\mathrm{T}_{h}^{0}-\mathrm{T}_{h}\right) v^{h}\right\|_{V} .
$$

Using the continuity of $\left(\mathrm{R}_{p}\right)_{p=1, P}$ and (26), one has for the first term of the right-hand side:

$$
\left\|\left(\mathrm{T}-\mathrm{T}_{h}^{0}\right) v^{h}\right\|_{V} \leq C \sum_{p=1, P}\left\|\chi_{p}-\chi_{p, h}\right\|_{W^{1, \infty}\left(\Omega_{2}\right)}\left\|v^{h}\right\|_{V} \leq C h\left\|v^{h}\right\|_{V} .
$$

On the other hand, for the last term of the right-hand side setting $w_{p, 2}^{h}=\mathrm{R}_{p} v_{1}^{h}, p=1, P$, one finds

$$
\left\|\left(\mathrm{T}_{h}^{0}-\mathrm{T}_{h}\right) v^{h}\right\|_{V} \leq 2 \sum_{p=1, P}\left\|\nabla\left(\chi_{p, h} w_{p, 2}^{h}-I_{h}\left(\chi_{p, h} w_{p, 2}^{h}\right)\right)\right\|_{\Omega_{2}^{h}}
$$

For every $p$, since the meshes are locally $\mathrm{T}$ conform, the discrete function $w_{p, 2}^{h}$ is equal to the restriction on $\Omega_{2}^{h}$ of an element of $V_{2}^{h}$. Using lemma 5 in $\S$ A.3 and the continuity of the operators $\left(\mathrm{R}_{p}\right)_{p=1, P}$, one finds

$$
\left\|\left(\mathrm{T}_{h}^{0}-\mathrm{T}_{h}\right) v^{h}\right\|_{V} \leq C h\left\|v^{h}\right\|_{V},
$$

which leads to (29), and thus to (28). It now follows that

$$
\exists \underline{\alpha}>0, \underline{\beta} \in \mathbb{R}, \forall h \text { small enough }, \forall v^{h} \in V^{h},\left|a\left(v^{h}, \mathrm{~T}_{h} v^{h}\right)\right| \geq\left(\underline{\alpha}-C_{0} h\right)\left\|v^{h}\right\|_{V}^{2}-\underline{\beta}\left\|\mathrm{C} v^{h}\right\|_{V}^{2},
$$

with $\underline{\alpha}$ and $\beta$ constants that express the weak T-coercivity of $a(\cdot, \cdot)$ with the operator $\mathrm{T}$ as in $(20)$-left, see lemma 2 and theorem 1 . Hence, one finds the desired result (27), which at the end yields the error estimate (cf. [11]).

Theorem 2. Assume that problem (1) is well-posed and that the meshes are locally T-conform. Then for $h$ small enough there exists one and only one solution $u^{h}$ to (4), with the estimate

$$
\left\|u-u^{h}\right\|_{V} \leq C \inf _{v^{h} \in V^{h}}\left\|u-v^{h}\right\|_{V}
$$

We conclude that we have optimality of the approximation for problem (1), thanks to the choice of the discrete T-coercivity operator as in (24), assuming only that the meshes are locally T-conform. 


\section{$6 \quad$ Numerical experiments}

We are now in position to present some numerical illustrations. Consider the case of a squared cavity with a triangular inclusion. Define the square $\Omega:=(-4 ; 4) \times(-4 ; 4): \Omega_{2}$ is an equilateral triangle of height 4 (the center of gravity $G$ of $\Omega_{2}$ is located at $\left(x_{1}, x_{2}\right)=(0,-1 / 3)$ ) and $\Omega_{1}=\Omega \backslash \overline{\Omega_{2}}$. We want to approximate problem (1) set in $\Omega$ with a constant right-hand side $f$ :

$$
\left\{\begin{array}{c}
\text { Find } u \in H_{0}^{1}(\Omega) \text { such that: } \\
-\operatorname{div}(\sigma \nabla u)=1 \text { in } \Omega
\end{array} .\right.
$$

Denoting $\left(c_{n}\right)_{n=1,3}$ the corners of $\Sigma$, then $\Omega_{1}$ locally coincides near $c_{n}, n=1,3$ with the cone of aperture $\alpha:=5 \pi / 3$. For this configuration one finds $I_{\alpha}:=5$, and with theorem 1 one can prove that if $\kappa_{\sigma} \notin[-5 ;-1 / 5]$ then problem (1) (with the data $f=1$ ) is well-posed in the Fredholm sense. Then in order to define locally T-conforming meshes, proceeding as in sections 3 and 5, one defines first a neighborhood of the interface $\Sigma$ made of three polygonal neighborhoods (one for each corner) where one applies the ad hoc symmetry-and rotation-based operator (21), and three trapezoid-based neighborhoods (one for each part edge) where one applies the symmetry-based operator (22). The associated locally T-conforming mesh is deduced by meshing one pattern of each neighborhood, and duplicating it by symmetry (see figure 8 (right)): in this configuration we choose $p_{1}=p_{2}=p_{3}=10$ and $q_{1}=q_{2}=q_{3}=2$.
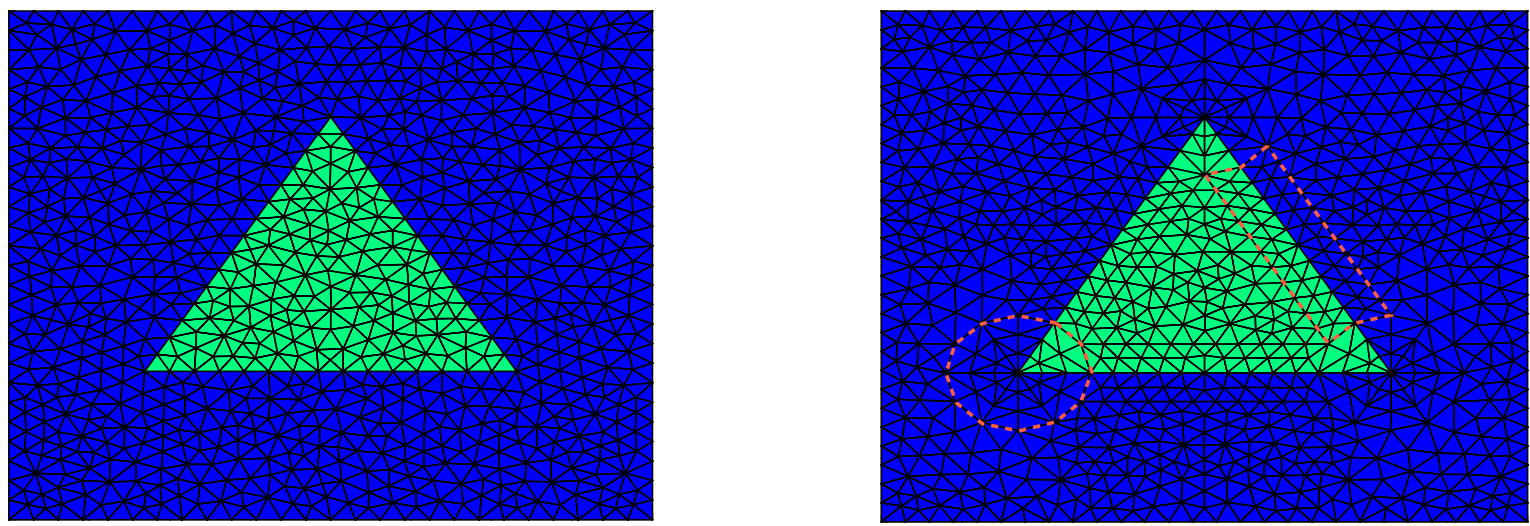

Figure 8: (Left) example a standard triangulation of $\Omega_{h}$ (1430 nodes) ; (right) example of locally T-conforming mesh (1424 nodes). The dashed lines enclose regions where we built T-conforming meshes near a corner, respectively a straight line.

In figure 9, we plot the relative errors in $L^{2}$-norm for several standard meshes (figure 8 (left)) and several T-conforming meshes (figure 8 (right)), for a contrast $\kappa_{\sigma}=-5.2$, and for several orders of approximation $(d=1,2,3)$. Note that we do not compare the discrete solution with the exact solution. As the optimality of the approximation (see theorem 2) with T-conforming meshes is satisfied, one can compare with $u_{h_{\min }}$ the discrete solution obtained on the finest mesh (with the same order of approximation) and one can check in figure 9 that convergence is ensured with a monotonic behavior of the error. With standard meshes however, the simulation gives unsatisfactory results with a slightly erratic behavior of the error. Numerical results illustrate the importance of using T-conforming meshes to guarantee convergence of the numerical method.

Due to the symmetry of the geometry (and the right-hand side), one is expecting a symmetric solution with respect to $\mathrm{Ox}_{2}$. One can check that, even for a refined, but standard mesh, the discrete solution does not satisfy this symmetry principle (see figure 10). This also explains why the approximation considering standard meshing does not converge so well.

On the other hand, according to [11, Proposition 2] the regularity of the solution $u$ of problem (1) is such that, away from the corners, $u_{i}, i=1,2$ is piecewise- $H^{2}$ whereas near the corners there exists a singularity exponent $s \in(0,1]$ such that $u_{i}$ is only guaranteed to be piecewise- $H^{1+s}$ due to the presence of a singular 


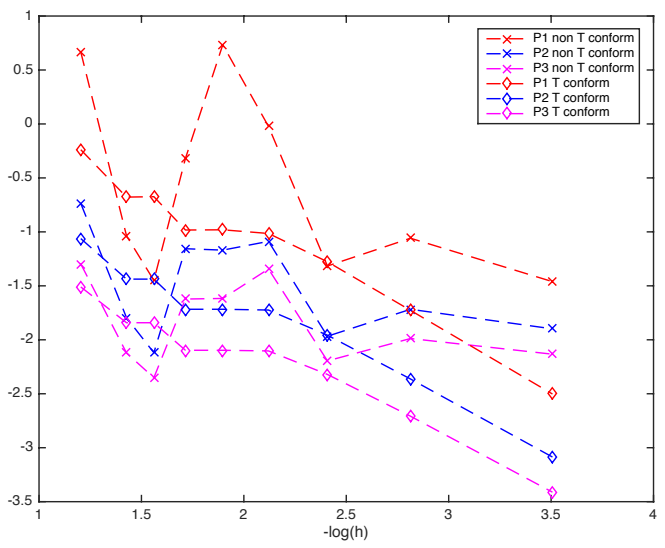

Figure 9: Relative error in $L^{2}$-norm of $u_{h}-u_{h_{\min }}$ for different mesh sizes $h$ (log-log scale) for $\kappa_{\sigma}=-5.2$.
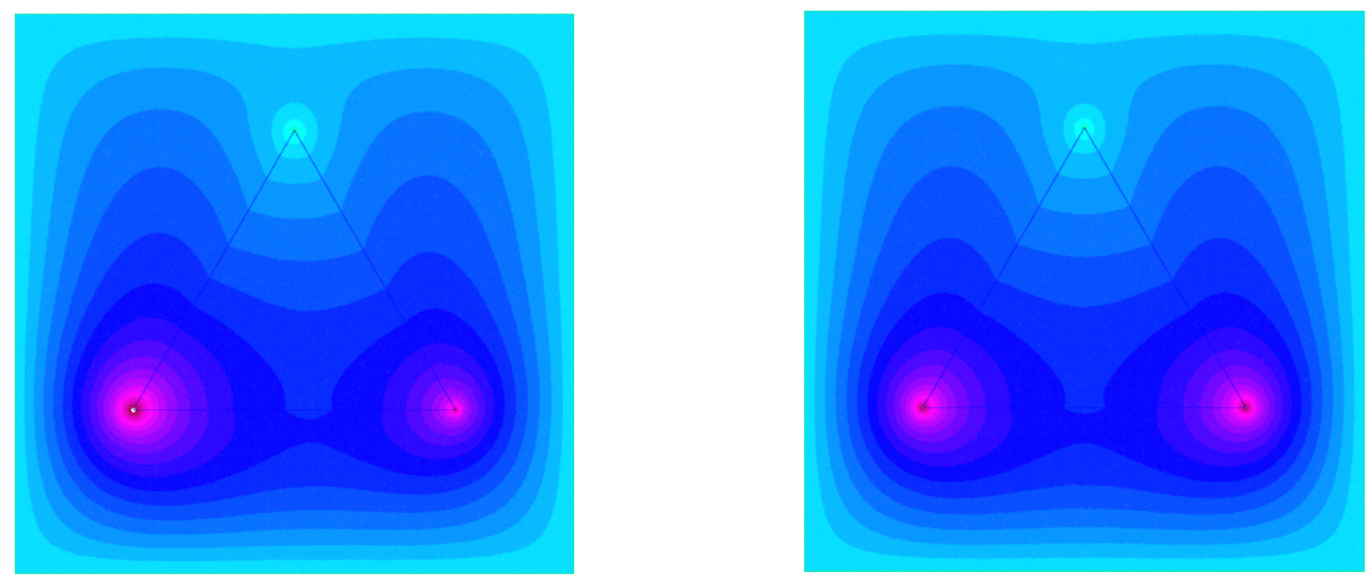

Figure 10: Computed solution, same color scale: (left) solution $u_{h}$ obtained with P3 finite elements on the finer standard mesh (101576 nodes); (right) solution $u_{h}$ obtained with P3 finite elements on the finer T-conforming mesh (101716 nodes).

part here (cf. section 3). Then one has the estimates

$$
\left\|u-u_{h}\right\|_{V} \leq C h^{s}, \quad \text { and } \quad\left\|u-u_{h}\right\|_{\Omega} \leq C h^{2 s} .
$$

The last estimate is obtained with the so-called Aubin-Nitsche lemma [16]. Note that in absence of the singular part, one expects that $s=1$. For $\kappa_{\sigma}=-5.2$ computations yield a singularity exponent $s=0.13$. The results of figure 9 show an average order of convergence equal to 0.9 (that is $s=0.45$ ) while using T-conforming meshes: this super-convergence phenomenon is classically observed when the reference solution is also a computed one, and both solutions are very close one to the other. On the other hand, using standard meshes, the average order of convergence for the last two meshes is $s=0.11$, which is almost equal to the theoretical value.

\section{Concluding remarks}

In this paper we extended the approximation theory for transmission problems with sign-changing coefficients with the T-coercivity approach. The novelty lies in the treatment of polygonal interfaces. This construction can be directly applied to the discrete problem by designing T-conforming meshes, which allows one to guarantee convergence of conforming finite element methods as soon as the continuous problem is well-posed. Below, we list some possible applications of our work. 
- One can study the transmission problem in the time-harmonic regime:

$$
\left\{\begin{array}{c}
\text { Find } u \in H_{0}^{1}(\Omega) \text { such that: } \\
\operatorname{div}(\sigma \nabla u)+\omega^{2} \varsigma u=f \text { in } \Omega
\end{array}\right.
$$

at the frequency $\omega \neq 0$, with $\varsigma \in L^{\infty}(\Omega)$. Indeed, the added term $\omega^{2} \varsigma u$ is a compact perturbation, so one concludes that when $\kappa_{\sigma} \notin\left[-I_{\alpha} ;-1 / I_{\alpha}\right]$, problem (31) is well-posed in the Fredholm sense and, when it is well-posed, one obtains convergence of the cFE method using locally T-conforming meshes.

- One can extend the results on problems (1) and (31) to the transmission problem with sign-changing coefficients and boundary conditions other than Dirichlet boundary condition (see for instance [10, Chapter 1, §1.7]).

- The previous proofs can be easily adapted to 2D geometries with a curved boundary or a curved interface. In particular, the case of the curved interface can be covered with the help of Theorem 3.1-1 of [13]. For an interface without corners, well-posedness in the Fredholm sense is then established as soon as $\kappa_{\sigma} \neq-1$ (see [8]). We refer to [15] for the first relation - and proof with the help of integral equations - of this result. Results for the optimality of the approximation hold using for instance isoparametric quadrilateral $\mathrm{FE}$ (see [8]).

- One may apply similar techniques to a transmission problem with sign-changing coefficients in 3D geometries with straight or curved boundary and interface. However the ability of the T-coercivity approach to deliver the optimal condition on the contrast has yet to be established in the general case [2].

- Once the well-posedness of problem (31) is established, one can solve the eigenvalue problem

$$
\left\{\begin{array}{l}
\text { Find }(u, \lambda) \in H_{0}^{1}(\Omega) \backslash\{0\} \times \mathbb{C} \text { such that: } \\
-\operatorname{div}(\sigma \nabla u)=\lambda u \text { in } \Omega
\end{array},\right.
$$

and derive classical error estimates for the eigenvalues' approximation (see $[8,9]$ ).

- Let us mention that a posteriori error estimates for diffusion problems with sign-changing coefficients have been carried out in [14] and lead to similar meshing requirements near the interfaces.

- On the other hand, the study of problem (1) when $\kappa_{\sigma}$ belongs to the critical interval cannot be handled as previously, due to the appearance of singularities $[20,6]$ that do not belong to $H^{1}(\Omega)$. This problem has been investigated in [4] for particular geometries: it requires a new functional framework explicitly taking into account the singularities. For a general polygonal interface, a new numerical approximation method is proposed in [3], which relies on the use of Perfectly Matched Layers at the corners.

\section{References}

[1] S. Anantha Ramakrishna, Physics of negative refractive index materials, Rep. Prog. Phys., 68 (2005), pp. $449-521$.

[2] A.-S. Bonnet-Ben Dhia, L. Chesnel, P. Ciarlet Jr., T-coercivity for scalar interface problems between dielectrics and metamaterials, Math. Mod. Num. Anal., 46 (2012), pp. 1363-1387.

[3] A.-S. Bonnet-Ben Dhia, C. Carvalho, L. Chesnel, P. Ciarlet Jr., On the use of Perfectly Matched Layers at corners for scattering problems with sign-changing coefficients, J. Comput. Phys., 322 (2016), pp. 224-247.

[4] A.-S. Bonnet-Ben Dhia, L. Chesnel, X. Claeys, Radiation condition for a non-smooth interface between a dielectric and a metamaterial, Math. Model. Meth. App. Sci., 23 (2013), pp. 1629-1662.

[5] A.-S. Bonnet-Ben Dhia, P. Ciarlet Jr., C.M. Zwölf, Time harmonic wave diffraction problems in materials with sign-shifting coefficients, J. Comput. Appl. Math., 234 (2010), pp. 1912-1919, Corrigendum p. 2616. 
[6] A.-S. Bonnet-Ben Dhia, M. Dauge, K. Ramdani, Analyse spectrale et singularités d'un problème de transmission non coercif, C. R. Acad. Sci. Paris, Ser. I, 328 (1999), pp. 717-720.

[7] H. Brezis, Functional analysis, Sobolev spaces and partial differential equations, Universitext, Springer, New York, 2011.

[8] C. Carvalho, Étude mathématique et numérique de structures plasmoniques avec des coins, Ph.D. thesis, École Polytechnique, 2015.

[9] C. Carvalho, L. Chesnel, P. Ciarlet Jr., Eigenvalue problems with sign-changing coefficients, C. R. Acad. Sci. Paris, Ser. I, 355 (2017), pp. 671-675.

[10] L. Chesnel, Étude de quelques problèmes de transmission avec changement de signe. Application aux métamatériaux, Ph.D. thesis, École Polytechnique, 2012.

[11] L. Chesnel, P. Ciarlet Jr., T-coercivity and continuous Galerkin methods: application to transmission problems with sign changing coefficients, Numer. Math., 124 (2013), pp. 1-29.

[12] E.T. Chung, P. Ciarlet Jr., A staggered discontinuous Galerkin method for wave propagation in media with dielectrics and meta-materials, J. Comput. Appl. Math., 239 (2013), pp. 189-207.

[13] P.G. Ciarlet, Mathematical Elasticity, Vol. III : Theory of Shells, North-Holland, Amsterdam, 2000.

[14] P. Ciarlet Jr., M. Vohralik, Robust a posteriori error control for transmission problems with sign-changing coefficients using localization of dual norms, hal-01148476, 2015.

[15] M. Costabel, E. Stephan, A direct boundary integral method for transmission problems, J. of Math. Anal. and Appl., 106 (1985), pp. 367-413.

[16] A. Ern, J.-L. Guermond, Theory and practice of finite elements, Springer-Verlag, Berlin, 2004.

[17] P. Grisvard, Singularities in boundary value problems, Masson, RMA 22, Paris, 1992.

[18] W. McLean, Strongly elliptic systems and boundary integral equations, Cambridge University Press, Cambridge, 2000.

[19] S. Nicaise, J. Venel, A posteriori error estimates for a finite element approximation of transmission problems with sign changing coefficients, J. Comput. Appl. Math., 235 (2011), pp. 4272-4282.

[20] K. Ramdani, Lignes supraconductrices : analyse mathématique et numérique, Ph.D. thesis, Université Paris 6, 1999.

[21] A.H. Schatz, An observation concerning Ritz-Galerkin methods with indefinite bilinear forms, Math. Comp., 28 (1974), pp. 959-962.

[22] D.R. Smith, J.B. Pendry, M.C.K. Wiltshire, Metamaterials and negative refractive index, Science, 305 (2004), pp. 788-792.

\section{A Appendix}

\section{A.1 General construction of $\mathrm{R}$ around corners}

In this section we generalize the tilings method presented in section 3 to any corner of angle $\alpha \in 2 \pi \mathbb{Q}$. Recall that we define $\alpha=2 \pi \frac{p}{p+q}$ in $\Omega_{1}$, with $p, q>0, p \neq q$ and $p+q$ even. Proceeding as in section 3 , one builds admissible rotation- and symmetry-based operators $\mathrm{R}^{\mathrm{adm}}$, and then take the average of these admissible operators to obtain the desired result (that is operators $R$ and $\mathrm{R}^{\prime}$ with the same minimal norm as in [2]). We propose $\min (p, q)$ admissible operators below based on a simple algorithm.

Consider for instance that $p<q$. One constructs $p$ admissible operators $\left(\mathrm{R}_{i}^{\text {adm }}\right)_{i=1, p}$ from $V_{1}$ to $V_{2}$, the $i$-th operator being obtained by (see figure 11 for an illustration): 
1) in $\Omega_{2}^{q+1-k}, k=1, i$ : apply $\mathrm{S}^{1}$ to $v_{1}^{k}$;

2) in $\Omega_{2}^{p+1-k}, k=i, p$ : apply $\mathrm{S}^{2}$ to $v_{1}^{k}$;

3) in $\Omega_{2}^{l}, l \in I:=\llbracket p+2-i, q-i \rrbracket$ : from $l=p+2-i$ to $l=q-i$, apply $\mathrm{R}_{i-(p+l)}$ to $v_{1}^{i}$, then $\mathrm{S}^{2} \circ \mathrm{R}_{p+1-i-(l+1)}$ to $v_{1}^{i}$, update $l \rightarrow l+2$ and so on. In other words, alternatively apply a rotation-based operator and a rotation+symmetry-based operator to $v_{1}^{i}$.

At step 3$),|I|=q-p-1$ is odd since $q+p$ is even, so one always finishes by $\mathrm{R}_{2 i-(p+q)}$ in $\Omega_{2}^{q+1-i}$, which ensures continuity of $\mathrm{R}_{i}^{\mathrm{adm}} v_{1}$ on $\partial \Omega_{2}^{q+1-i} \cap \partial \Omega_{2}^{q-i}$.

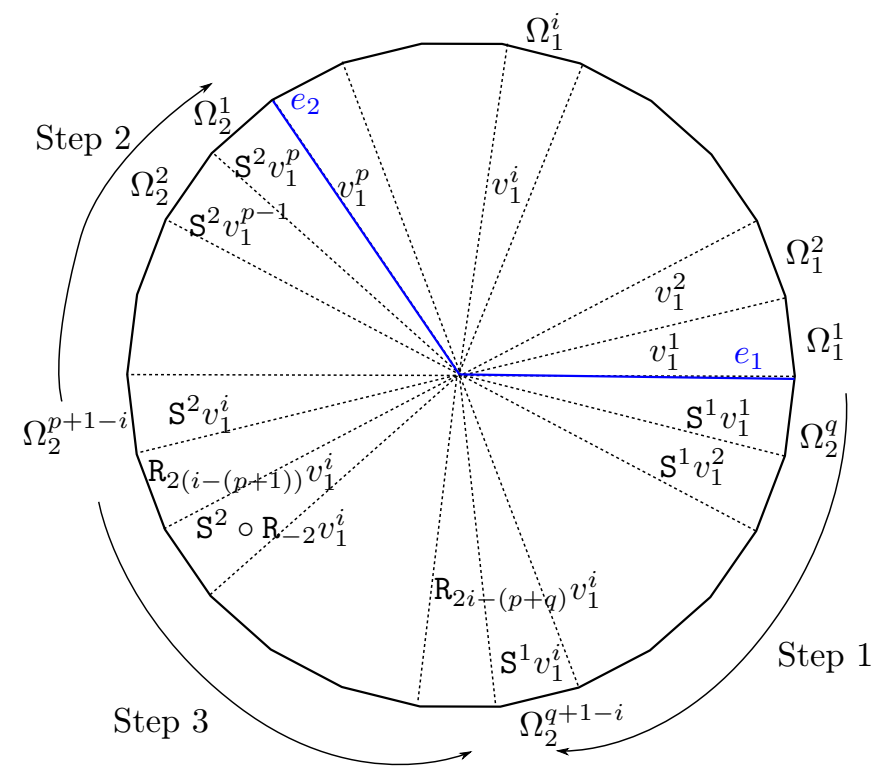

Figure 11: Scheme representing steps 1)-2)-3) for building $\mathrm{R}_{i}^{\mathrm{adm}}$.

One constructs $p$ admissible operators $\left(\mathrm{R}_{i}^{\text {adm }}\right)_{i=1, p}$ from $V_{2}$ to $V_{1}$ similarly, the $i$-th operator being obtained by:

4) in $\Omega_{1}^{k}, k=1, \min (i, p-1)$ : apply $\mathrm{S}^{1}$ to $v_{2}^{q+1-k}$;

5) in $\Omega_{1}^{k}, k=\min (i+1, p), p$ : apply $\mathrm{S}^{2}$ to $v_{2}^{p+1-k}$;

6) in $\Omega_{1}^{i}$, if $i<p$, add up the remaining contributions $\left(v_{2}^{l}\right)_{l \in I^{\prime}}$, with $I^{\prime}:=\llbracket p+1-i, q-i \rrbracket$ : for $l=p+1-i$ to $l=q-i$, start by $\mathrm{S}^{2} \circ \mathrm{R}_{l-(p+1-i)}$ then apply $-\mathrm{R}_{(l+1)+p-i}$, update $l \rightarrow l+2$ and so on. If $i=p$ one adds up $\left(v_{2}^{l}\right)_{l \in I^{\prime}}$, where $I^{\prime}=\llbracket 2, q-p+1 \rrbracket$ : for $l=2$ to $l=q-p+1$ start with $-\mathrm{R}_{l}$ then apply the rotation + symmetry-based operator $\mathrm{S}^{1} \circ \mathrm{R}_{(q-p+1)-(l+1)}$, update $l \rightarrow l+2$ and so on.

At step 6), in both cases, $\left|I^{\prime}\right|=q-p$ is even since $q+p$ is even, so one applies successively pairs of operators. Note also that at step 6), one adds contributions in the $i$-th pattern of $\Omega_{1}$, already considered at step 4) or 5 ), and remark that when the index is zero, $R_{0}=I$ so that the rotation+symmetry-based operator simply becomes a symmetry-based operator.

Since $p+q$ is even, one can check that continuity is ensured at all interfaces of the patterns. The algorithm produces $p$ operators from $V_{1}$ to $V_{2}$, respectively from $V_{2}$ to $V_{1}$. Let us give an example: for $p=4, q=6$ (that is $\alpha=4 \pi / 5$ ). 
Then one finds four admissible operators from $V_{1}$ to $V_{2}$ (each one corresponds to a column):

$\begin{array}{ccccc}\mathrm{R}_{1}^{\operatorname{adm}} v_{1} & \mathrm{R}_{2}^{\mathrm{adm}} v_{1} & \mathrm{R}_{3}^{\mathrm{adm}} v_{1} & \mathrm{R}_{4}^{\mathrm{adm}} v_{1} & \\ \text { "I } & \text { "l } & \text { "I } & \text { " } & \\ \mathrm{S}^{2} v_{1}^{4}, & \mathrm{~S}^{2} v_{1}^{4}, & \mathrm{~S}^{2} v_{1}^{4}, & \mathrm{~S}^{2} v_{1}^{4}, & \text { in } \Omega_{2}^{1} \\ \mathrm{~S}^{2} v_{1}^{3}, & \mathrm{~S}^{2} v_{1}^{3}, & \mathrm{~S}^{2} v_{1}^{3}, & \mathrm{R}_{-2} v_{1}^{4}, & \text { in } \Omega_{2}^{2} \\ \mathrm{~S}^{2} v_{1}^{2}, & \mathrm{~S}^{2} v_{1}^{2}, & \mathrm{R}_{-4} v_{1}^{3}, & \mathrm{~S}^{1} v_{1}^{4}, & \text { in } \Omega_{2}^{3} \\ \mathrm{~S}^{2} v_{1}^{1}, & \mathrm{R}_{-6} v_{1}^{2}, & \mathrm{~S}^{1} v_{1}^{3}, & \mathrm{~S}^{1} v_{1}^{3}, & \text { in } \Omega_{2}^{4} \\ \mathrm{R}_{-8} v_{1}^{1}, & \mathrm{~S}^{1} v_{1}^{2}, & \mathrm{~S}^{1} v_{1}^{2}, & \mathrm{~S}^{1} v_{1}^{2}, & \text { in } \Omega_{2}^{5} \\ \mathrm{~S}^{1} v_{1}^{1}, & \mathrm{~S}^{1} v_{1}^{1}, & \mathrm{~S}^{1} v_{1}^{1}, & \mathrm{~S}^{1} v_{1}^{1}, & \text { in } \Omega_{2}^{6}\end{array}$

In this example, $|I|=1$ so one applies only one rotation-based operator (following step 3)). Conversely, one finds four admissible operators from $V_{2}$ to $V_{1}$ :

$$
\begin{array}{ccccc}
\mathrm{R}_{1}^{\mathrm{adm}}{ }^{\prime} v_{2} & \mathrm{R}_{2}^{\mathrm{adm} m^{\prime}} v_{2} & \mathrm{R}_{3}^{\mathrm{adm} m^{\prime}} v_{2} & \mathrm{R}_{4}^{\mathrm{adm}} v_{2} & \\
\| & \| & \| & \| 1 & \\
\mathrm{~S}^{1} v_{2}^{6}-\mathrm{R}_{8} v_{2}^{5}+\mathrm{S}^{2} v_{2}^{4}, & \mathrm{~S}^{1} v_{2}^{6}, & \mathrm{~S}^{1} v_{2}^{6}, & \mathrm{~S}_{2}^{6}, & \text { in } \Omega_{1}^{1} \\
\mathrm{~S}^{2} v_{2}^{3}, & \mathrm{~S}^{1} v_{2}^{5}-\mathrm{R}_{6} v_{2}^{4}+\mathrm{S}^{2} v_{2}^{3}, & \mathrm{~S}^{1} v_{2}^{5}, & \mathrm{~S}^{1} v_{2}^{5}, & \text { in } \Omega_{1}^{2} \\
\mathrm{~S}^{2} v_{2}^{2}, & \mathrm{~S}^{2} v_{2}^{2}, & \mathrm{~S}^{1} v_{2}^{4}-\mathrm{R}_{4} v_{2}^{3}+\mathrm{S}^{2} v_{2}^{2}, & \mathrm{~S}^{1} v_{2}^{4}, & \text { in } \Omega_{1}^{3} \\
\mathrm{~S}^{2} v_{2}^{1}, & \mathrm{~S}^{2} v_{2}^{1}, & \mathrm{~S}^{2} v_{2}^{1}, & \mathrm{~S}^{2} v_{2}^{1}-\mathrm{R}_{2} v_{2}^{2}+\mathrm{S}^{1} v_{2}^{3}, & \text { in } \Omega_{1}^{4}
\end{array}
$$

Here $\left|I^{\prime}\right|=2$ so one applies two additional operators to $v_{2}^{l}, l \in I^{\prime}$ (following step 6)).

With these guidelines one can write all operators $\left(\mathrm{R}_{i}^{\mathrm{adm}}\right)_{i=1, p},\left(\mathrm{R}_{i}^{\mathrm{adm}}\right)_{i=1, p}$ for any $p, q$ such that $p<q$ and $p+q$ even. Note that by exchanging $p$ with $q$ (and $\Omega_{1}$ with $\Omega_{2}$ ), one addresses similarly the case $q<p$.

In the following, we set $p<q$. As mentioned in section 3, the problem is that, taken individually, no admissible operator $\mathrm{R}_{i}^{\text {adm }}\left(\right.$ resp. $\mathrm{R}_{i}^{\text {adm }}$ ), $i=1, p$, satisfies $\left\|\mathrm{R}_{i}^{\text {adm }}\right\|^{2}=I_{\alpha}\left(\right.$ resp. $\left.\left\|\mathrm{R}_{i}^{\mathrm{adm}^{\prime}}\right\|^{2}=I_{\alpha}\right)$, with $I_{\alpha}$ defined in (9). Indeed, for all $v_{1} \in V_{1}, v_{2} \in V_{2}$, for $i=1, p, \mathrm{R}_{i}^{\text {adm }}$ built from 1)-2)-3) and $\mathrm{R}_{i}^{\text {adm }}{ }^{\prime}$ built from 4)-5)-6), one gets the bounds

$$
\begin{gathered}
\left\|\nabla\left(\mathrm{R}_{i}^{\mathrm{adm}} v_{1}\right)\right\|_{\Omega_{2}}^{2} \leq \sum_{k=1, p}\left\|\nabla v_{1}^{k}\right\|_{\Omega_{1}^{k}}^{2}+(|I|+1)\left\|\nabla v_{1}^{i}\right\|_{\Omega_{1}^{i}}^{2} \leq(q-p+1)\left\|\nabla v_{1}\right\|_{\Omega_{1}}^{2}, \\
\left\|\nabla\left(\mathrm{R}_{i}^{\mathrm{adm}^{\prime}} v_{2}\right)\right\|_{\Omega_{1}}^{2} \leq \sum_{l \in \llbracket 1, q \rrbracket \backslash I^{\prime}}\left\|\nabla v_{2}^{l}\right\|_{\Omega_{2}^{l}}^{2}+\left(\left|I^{\prime}\right|+1\right) \sum_{l \in I^{\prime}}\left\|\nabla v_{2}^{l}\right\|_{\Omega_{2}^{l}}^{2} \leq(q-p+1)\left\|\nabla v_{2}\right\|_{\Omega_{2}}^{2} .
\end{gathered}
$$

The bounds are sharp since:

- in the first case, one may choose $v_{1} \neq 0$ such that $v_{1}^{k}=0$ for $k \neq i$ : then $\left\|\nabla\left(\mathrm{R}_{i}^{\text {adm }} v_{1}\right)\right\|_{\Omega_{2}}^{2}=(q-p+$ 1) $\left\|\nabla v_{1}\right\|_{\Omega_{1}}^{2}$;

- in the second case, given $l \in I^{\prime}(\neq \emptyset)$, one may choose $v_{2} \neq 0$ such that $v_{2}^{k}=0$ for $k \neq l$ : then $\left\|\nabla\left(\mathrm{R}_{i}^{\mathrm{adm}^{\prime}} v_{2}\right)\right\|_{\Omega_{1}}^{2}=(q-p+1)\left\|\nabla v_{2}\right\|_{\Omega_{2}}^{2}$.

One can check that $I_{\alpha}<q-p+1$ for all $1<p<q$ : $I_{\alpha}-(q-p+1)=(p-q)(p-1) / p<0\left({ }^{9}\right)$.

To get optimal operators (that is of norm equal to $I_{\alpha}$ ), one defines $\mathrm{R}, \mathrm{R}^{\prime}$ as the average of the admissible operators:

$$
\mathrm{R}=\frac{1}{p} \sum_{i=1, p} \mathrm{R}_{i}^{\mathrm{adm}}, \quad \text { and } \quad \mathrm{R}^{\prime}=\frac{1}{p} \sum_{i=1, p} \mathrm{R}_{i}^{\text {adm }}
$$

\footnotetext{
${ }^{9}$ Equality is obtained for $p=q$ (corresponding to a plane interface) for which $\left\|\mathrm{R}_{i}^{\text {adm }}\right\|^{2}=\left\|\mathrm{R}_{i}^{\text {adm }}{ }^{\prime}\right\|^{2}=1$, or $p=1$ : in that case there is only one admissible operator.
} 
Going back to our example with $p=4$ and $q=6$, one defines $\mathrm{R}$ and $\mathrm{R}^{\prime}$ such that for all $v_{1} \in V_{1}, v_{2} \in V_{2}$

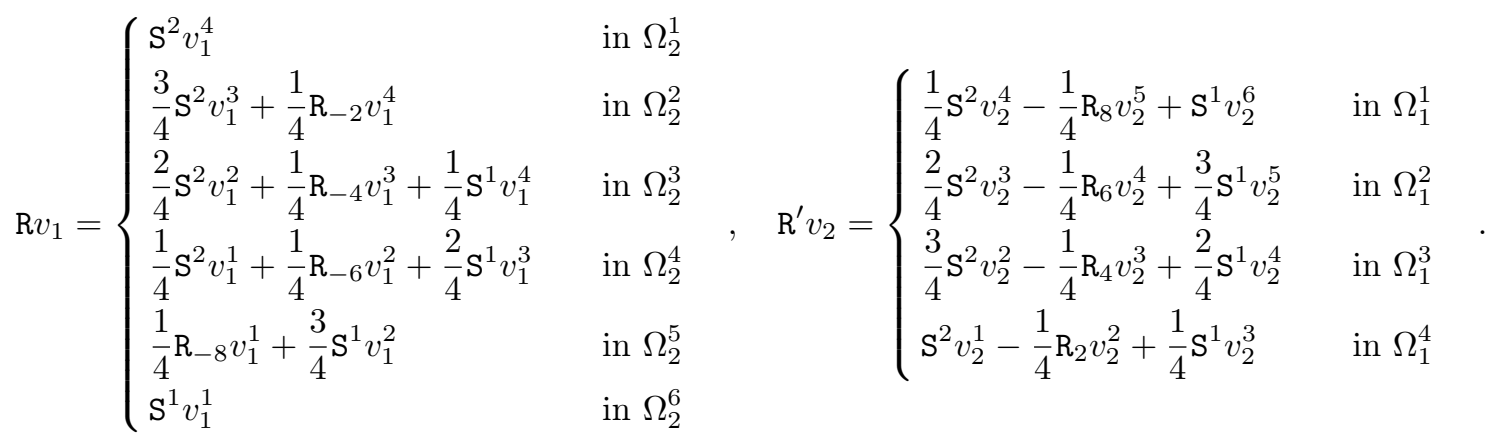

These operators are optimal in the sense that $\|\mathrm{R}\|^{2}=\left\|\mathrm{R}^{\prime}\right\|^{2}=I_{\alpha}$. Proceeding as in section 3, using the triangle inequality one finds that, for all $v_{1} \in V_{1}$,

$$
\begin{aligned}
\left\|\nabla\left(\mathrm{R} v_{1}\right)\right\|_{\Omega_{2}}^{2} & \leq\left\|\nabla v_{1}^{4}\right\|_{\Omega_{1}^{4}}^{2}+\left(\frac{3}{4}\left\|\nabla v_{1}^{3}\right\|_{\Omega_{1}^{3}}+\frac{1}{4}\left\|\nabla v_{1}^{4}\right\|_{\Omega_{1}^{4}}\right)^{2}+\left(\frac{2}{4}\left\|\nabla v_{1}^{2}\right\|_{\Omega_{1}^{2}}+\frac{1}{4}\left\|\nabla v_{1}^{3}\right\|_{\Omega_{1}^{3}}+\frac{1}{4}\left\|\nabla v_{1}^{4}\right\|_{\Omega_{1}^{4}}\right)^{2} \\
& +\left(\frac{1}{4}\left\|\nabla v_{1}^{1}\right\|_{\Omega_{1}^{1}}+\frac{1}{4}\left\|\nabla v_{1}^{2}\right\|_{\Omega_{1}^{2}}+\frac{2}{4}\left\|\nabla v_{1}^{3}\right\|_{\Omega_{1}^{3}}\right)^{2}+\left(\frac{1}{4}\left\|\nabla v_{1}^{1}\right\|_{\Omega_{1}^{1}}+\frac{3}{4}\left\|\nabla v_{1}^{2}\right\|_{\Omega_{1}^{2}}\right)^{2}+\left\|\nabla v_{1}^{1}\right\|_{\Omega_{1}^{1}}^{2}
\end{aligned}
$$

By defining the matrix $M=\left(\begin{array}{cccc}0 & 0 & 0 & 1 \\ 0 & 0 & 3 / 4 & 1 / 4 \\ 0 & 2 / 4 & 1 / 4 & 1 / 4 \\ 1 / 4 & 1 / 4 & 2 / 4 & 0 \\ 1 / 4 & 3 / 4 & 0 & 0 \\ 1 & 0 & 0 & 0\end{array}\right)$, and $\vec{W}:=\left(\left\|\nabla v_{1}^{1}\right\|_{\Omega_{1}^{1}},\left\|\nabla v_{1}^{2}\right\|_{\Omega_{1}^{2}},\left\|\nabla v_{1}^{3}\right\|_{\Omega_{1}^{3}},\left\|\nabla v_{1}^{4}\right\|_{\Omega_{1}^{4}}\right)^{\top}$, one can check that $\left\|\nabla\left(\mathrm{R} v_{1}\right)\right\|_{\Omega_{2}}^{2} \leq\|M \vec{W}\|_{2}^{2} \leq\left\|M^{\top} M\right\|_{2}\left\|\nabla v_{1}\right\|_{\Omega_{1}}^{2}$, leading to $\|\mathrm{R}\|^{2} \leq\left\|M^{\top} M\right\|_{2}$. Similarly, by defining $M^{\prime}=M^{\top}$, one can check that $\left\|\mathrm{R}^{\prime}\right\|^{2} \leq\left\|M^{\prime \top} M^{\prime}\right\|_{2}$.

Let us remark that the entries $M_{l k}, l=1,6, k=1,4$, are such that $M_{l k}=\sup _{w_{1} \in V_{1}} \frac{\left\|\nabla\left(\mathrm{R} w_{1}\right)\right\|_{\Omega_{2}^{l}}}{\left\|\nabla w_{1}^{k}\right\|_{\Omega_{1}^{k}}}$. For example for $l=4$, one has for all $v_{1} \in V_{1}$,

$$
\left\|\nabla\left(\mathrm{R} v_{1}\right)\right\|_{\Omega_{2}^{4}}^{2} \leq\left(\frac{1}{4}\left\|\nabla v_{1}^{1}\right\|_{\Omega_{1}^{1}}+\frac{1}{4}\left\|\nabla v_{1}^{2}\right\|_{\Omega_{1}^{2}}+\frac{2}{4}\left\|\nabla v_{1}^{3}\right\|_{\Omega_{1}^{3}}\right)^{2} .
$$

One obtains equality by taking, for every $k=1,4, v_{1}$ such that $v_{1}^{i}=0$ for $i \neq k$, which yields $M_{41}=\frac{1}{4}$, $M_{42}=\frac{1}{4}, M_{43}=\frac{2}{4}, M_{44}=0$.

More generally, one obtains for instance for $v_{1} \in V_{1}$

$$
\left\|\nabla\left(\mathrm{R} v_{1}\right)\right\|_{\Omega_{2}}^{2}=\sum_{l=1, q}\left\|\nabla\left(\mathrm{R} v_{1}\right)\right\|_{\Omega_{2}^{l}}^{2} \leq \sum_{l=1, q}\left(\sum_{k=1, p} M_{l k}\left\|\nabla v_{1}^{k}\right\|_{\Omega_{1}^{k}}\right)^{2}, \text { with } M_{l k}=\sup _{w_{1} \in V_{1}} \frac{\left\|\nabla\left(\mathrm{R} w_{1}\right)\right\|_{\Omega_{2}^{l}}}{\left\|\nabla w_{1}^{k}\right\|_{\Omega_{1}^{k}}} .
$$

Above, a sum over $k$ appears due to the fact that for $l=1, q$, the $\left.\left(\mathrm{R}_{i}^{\mathrm{adm}} v_{1}\right)\right|_{\Omega_{2}^{l}}$ is a linear combination of isometry-based operators applied to some $v_{1}^{k}$. Introducing $M \in \mathcal{M}_{q, p}(\mathbb{R})$ with entries $\left(M_{l k}\right)_{l=1, q, k=1, p}$, one has (see footnote 3 p. 6)

$$
\|\mathrm{R}\|^{2}=\left\|M^{\top} M\right\|_{2} .
$$


Let us give the general expression of the matrix $M$ for any $p, q$, and evaluate $\left\|M^{\top} M\right\|_{2}$ to conclude. For $n \in \llbracket 1, p \rrbracket$, define $M_{p-n+1}, \widetilde{M}_{p-n+1} \in \mathcal{M}_{p-n+1, p}(\mathbb{R})$ such that

$$
\begin{aligned}
& M_{p-n+1}
\end{aligned}
$$

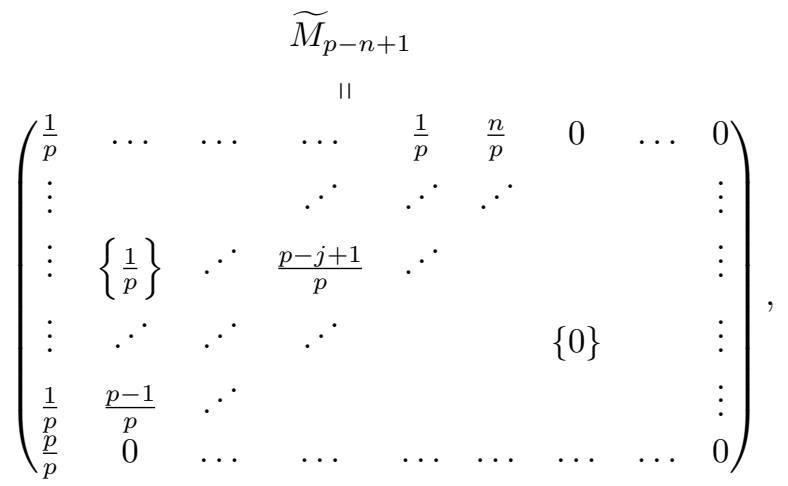

$$
\begin{aligned}
& \text { II } \\
& \left(\begin{array}{ccccccccc}
0 & \ldots & \ldots & \ldots & \ldots & \ldots & \ldots & 0 & \frac{p}{p} \\
\vdots & & & & & & . & \frac{p-1}{p} & \frac{1}{p} \\
\vdots & & \{0\} & & & . \cdot & . & . & \vdots \\
\vdots & & & & . \cdot & \frac{j}{p} & . & \left\{\frac{1}{p}\right\} & \vdots \\
\vdots & & & . \cdot & . \cdot & . \cdot & & & \vdots \\
0 & \ldots & 0 & \frac{n}{p} & \frac{1}{p} & \ldots & \ldots & \ldots & \frac{1}{p}
\end{array}\right)
\end{aligned}
$$

where $j$ denotes the column index. In the specific case where $n=1$, the matrix $M_{p}$ (resp. $\widetilde{M}_{p}$ ) is a square matrix for which the first column (resp. last column) has non zero entries. All entries are equal to 0 or $\frac{1}{p}$, except on one diagonal where they range from $\frac{n}{p}$ to $\frac{p}{p}=1$. Then the shape of $M$ depends on whether $p \leq \frac{q}{2}$ or not:

- if $p \leq q / 2$ : let $m \in \mathbb{N}$ such that $q=2 p+m$. Then the matrix $M$ is written $M=\left(\begin{array}{l}M_{p} \\ M_{m}^{\prime} \\ \widetilde{M}_{p}\end{array}\right)$ with $M_{p}, \widetilde{M}_{p} \in \mathcal{M}_{p}(\mathbb{R})$ (defined above for $n=1$ ) and respectively $M_{m}^{\prime} \in \mathcal{M}_{m, p}(\mathbb{R})$ whose entries are all equal to $1 / p$, with the convention that if $m=0, M_{m}^{\prime}$ is empty. The example treated in section 3 corresponds to that case with $m=0$.

- if $p>q / 2$ : let $m \in \mathbb{N}^{*}$ such that $q=2 p-m$. If $m>2$ then the matrix $M$ is written $M=\left(\begin{array}{c}M_{p-m+1} \\ M_{m-2}^{\prime \prime} \\ \widetilde{M}_{p-m+1}\end{array}\right)$, with $M_{p-m+1}, \widetilde{M}_{p-m+1} \in \mathcal{M}_{p-m+1, p}(\mathbb{R})$ (defined above for $n=m$ ) and resp. $M_{m-2}^{\prime \prime} \in \mathcal{M}_{m-2, p}(\mathbb{R})$ such that (below $i$ denotes the row index)

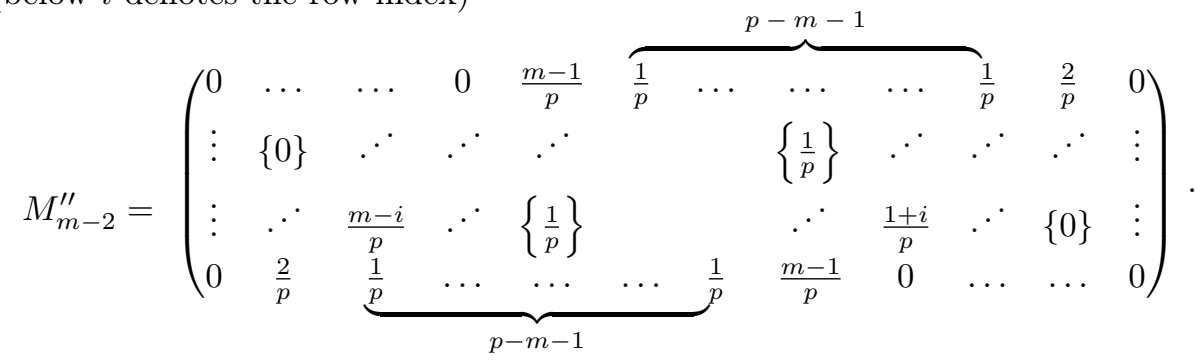

It is convenient to define $M_{m-2}^{\prime \prime}$ according to some $d \in \mathbb{Z}$, defined such that $p=2(m-2)+d$. For $d>1$, one can obtain some "central" columns with only $\frac{1}{p}$ entries, whereas for $d \leq 1$, one gets central columns of the form $\left(0, \ldots, 0, \frac{m-j}{p}, \frac{1}{p}, \ldots, \frac{1}{p}, \frac{1+i}{p}, 0, \ldots, 0\right)^{\top}$ and $\left(0, \ldots, 0, \frac{1+j}{p}, \frac{1}{p}, \ldots, \frac{1}{p}, \frac{m-i}{p}, 0, \ldots, 0\right)^{\top}$ for some ad hoc $i, j$ that govern the number of 0 entries. For example for $d=0$ the two central columns are given by $\left(\frac{m-1}{p}, \frac{1}{p}, \ldots, \frac{1}{p}, \frac{m-2}{p}, 0\right)^{\top},\left(0, \frac{m-2}{p}, \frac{1}{p}, \ldots, \frac{1}{p}, \frac{m-1}{p}\right)^{\top}$ and for $d=1$ the central column is $\left(\frac{m-1}{p}, \frac{1}{p}, \ldots, \frac{1}{p}, \frac{m-1}{p}\right)^{\top}$. The key is to start with the first and the last rows, then complete the rest accordingly.

The matrix $M$ simplifies to the matrix $M=\left(\begin{array}{c}M_{p-1} \\ \frac{1}{p} \ldots \frac{1}{p} \\ \widetilde{M}_{p-1}\end{array}\right)$ when $q=2 p-1$, and to the matrix $M=$ $\left(\begin{array}{l}M_{p-1} \\ \widetilde{M}_{p-1}\end{array}\right)$ when $q=2 p-2$. The previous example with $p=4, q=6$ corresponds to that last case. 
Note that the cases $p>q$ are obtained by taking the transpose of the matrix presented above.

Let us give two examples to illustrate the cases $p \leq q / 2$ and $p>q / 2$.

Case $p=2, q=6$ : in that case $q=2 p+m$ with $m=2$. Following 1)-2)-3) and using (33)-(34), one can check that for $v_{1} \in V_{1}$,

$$
\mathrm{R} v_{1}=\left\{\begin{array}{ll}
\mathrm{S}^{2} v_{1}^{2} & \text { in } \Omega_{2}^{1} \\
\frac{1}{2} \mathrm{~S}^{2} v_{1}^{1}+\frac{1}{2} \mathrm{R}_{-2} v_{1}^{2} & \text { in } \Omega_{2}^{2} \\
\frac{1}{2} \mathrm{R}_{-4}^{2} v_{1}^{1}+\frac{1}{2} \mathrm{~S}^{2} \circ \mathrm{R}_{-2} v_{1}^{2} & \text { in } \Omega_{2}^{3} \\
\frac{1}{2} \mathrm{~S}^{2} \circ \mathrm{R}_{-4} v_{1}^{1}+\frac{1}{2} \mathrm{R}_{-4} v_{1}^{2} & \text { in } \Omega_{2}^{4} \\
\frac{1}{2} \mathrm{R}_{-6} v_{1}^{1}+\frac{1}{2} \mathrm{~S}^{1} v_{1}^{2} & \text { in } \Omega_{2}^{5} \\
\mathrm{~S}^{1} v_{1}^{1} & \text { in } \Omega_{2}^{6}
\end{array} \quad, \quad \text { and } M=\left(\begin{array}{cc}
0 & 1 \\
1 / 2 & 1 / 2 \\
1 / 2 & 1 / 2 \\
1 / 2 & 1 / 2 \\
1 / 2 & 1 / 2 \\
1 & 0
\end{array}\right)=\left(\begin{array}{l}
M_{2} \\
M_{2}^{\prime} \\
\widetilde{M}_{2}
\end{array}\right) .\right.
$$

Case $p=6, q=8$ : in that case $q=2 p-m$ with $m=4$. One can check that for $v_{1} \in V_{1}$,

$$
\mathrm{R} v_{1}= \begin{cases}\mathrm{S}^{2} v_{1}^{6} & \text { in } \Omega_{2}^{1} \\ \frac{5}{6} \mathrm{~S}^{2} v_{1}^{5}+\frac{1}{6} \mathrm{R}_{-2} v_{1}^{6} & \text { in } \Omega_{2}^{2} \\ \frac{4}{6} \mathrm{~S}^{2} v_{1}^{4}+\frac{1}{6} \mathrm{R}_{-4}^{2} v_{1}^{5}+\frac{1}{6} \mathrm{~S}^{2} v_{1}^{6} & \text { in } \Omega_{2}^{3} \\ \frac{3}{6} \mathrm{~S}^{2} v_{1}^{3}+\frac{1}{6} \mathrm{R}_{-6}^{2} v_{1}^{4}+\frac{2}{6} \mathrm{~S}^{2} v_{1}^{5} & \text { in } \Omega_{2}^{4} \\ \frac{2}{6} \mathrm{~S}^{2} v_{1}^{2}+\frac{1}{6} \mathrm{R}_{-8}^{2} v_{1}^{3}+\frac{3}{6} \mathrm{~S}^{2} v_{1}^{4} & \text { in } \Omega_{2}^{5} \\ \frac{1}{6} \mathrm{~S}^{2} v_{1}^{1}+\frac{1}{6} \mathrm{R}_{-10}^{2} v_{1}^{2}+\frac{4}{6} \mathrm{~S}^{2} v_{1}^{3} & \text { in } \Omega_{2}^{6} \\ \frac{1}{6} \mathrm{R}_{-12} v_{1}^{1}+\frac{5}{6} \mathrm{~S}^{2} v_{1}^{2} & \text { in } \Omega_{2}^{7} \\ \mathrm{~S}^{1} v_{1}^{1} & \text { in } \Omega_{2}^{8}\end{cases}
$$

Moreover, one checks easily by direct inspection the next result.

Proposition 1. The matrices $M_{p-n+1} \in \mathcal{M}_{p-n+1, p}(\mathbb{R}), \widetilde{M}_{p-n+1} \in \mathcal{M}_{p-n+1, p}(\mathbb{R}), M_{m}^{\prime} \in \mathcal{M}_{m, p}(\mathbb{R}), M_{m-2}^{\prime \prime} \in$ $\mathcal{M}_{m-2, p}(\mathbb{R})$ satisfy the following properties:

$$
\begin{aligned}
& \text { 1) } \sum_{l=1}^{p-n+1}\left(M_{p-n+1}\right)_{l k}=\left\{\begin{array}{ll}
0 & \text { if } k<n \\
\frac{2 k-n}{p} & \text { if } k \geq n
\end{array} ; \quad \forall l=1, p-n+1, \sum_{k=1}^{p}\left(M_{p-n+1}\right)_{l k}=1 ;\right. \\
& \text { 2) } \quad \sum_{l=1}^{p-n+1}\left(\widetilde{M}_{p-n+1}\right)_{l k}=\left\{\begin{array}{ll}
\frac{2(p+1-k)-n}{p} & \text { if } k \leq p-n+1 \\
0 & \text { if } k>p-n+1
\end{array} ; \quad \forall l=1, p-n+1, \sum_{k=1}^{p}\left(\widetilde{M}_{p-n+1}\right)_{l k}=1 ;\right. \\
& \text { 3) } \forall k=1, p, \sum_{l=1}^{m}\left(M_{m}^{\prime}\right)_{l k}=\frac{m}{p} ; \quad \forall l=1, m, \sum_{k=1}^{p}\left(M_{m}^{\prime}\right)_{l k}=1 \text {; } \\
& \text { 4) } \sum_{l=1}^{m-2}\left(M_{m-2}^{\prime \prime}\right)_{l k}=\left\{\begin{array}{ll}
\frac{2(k-1)}{p} & \text { if } k<\min (m, m-2+d) \\
\frac{m-2}{p} & \text { if } m \leq k \leq p-m+1 \quad \text { for } d>1 \\
\frac{q}{p} & \text { if } m-2+d \leq k \leq p-m+3-d \quad \text { for } d \leq 1 \\
\frac{2(p-k)}{p} & \text { if } \max (p-m+1, p-m+3-d)<k
\end{array}\right. \text {; }
\end{aligned}
$$




$$
\forall l=1, m-2, \sum_{k=1}^{p}\left(M_{m-2}^{\prime \prime}\right)_{l k}=1
$$

Proposition 2. The matrix $M \in \mathcal{M}_{q, p}(\mathbb{R})$ satisfies the following properties:

[1] $\left\{\begin{array}{l}\forall l=1, q, \forall k=1, p, M_{l k} \geq 0 \\ \exists l_{0}, k_{0} \text { s.t. } M_{l_{0} k_{0}}=1\end{array} \quad ;[2] \forall l,\left\{\begin{array}{l}\sum_{k=1}^{p} M_{l k}=1 \quad \text { if } p \leq q \\ \sum_{k=1}^{p} M_{l k}=\frac{p}{q} \quad \text { if } p \geq q\end{array} \quad ;[3] \forall k,\left\{\begin{array}{l}\sum_{l=1}^{q} M_{l k}=\frac{q}{p} \quad \text { if } p \leq q \\ \sum_{l=1}^{q} M_{l k}=1 \quad \text { if } p \geq q\end{array}\right.\right.\right.$.

Proof. Using proposition 1 one can deduce all the above results. Let us simply detail how to obtain the first result of [3], i.e. when $q \geq p$. First consider the case where $q=2 p+m, m \in \llbracket 1, p \rrbracket$. Then for all $k=1, p$ one has

$$
\sum_{l=1}^{q} M_{l k}=\sum_{l=1}^{p}\left(M_{p}\right)_{l k}+\sum_{l=1}^{m}\left(M_{m}^{\prime}\right)_{l k}+\sum_{l=1}^{p}\left(\widetilde{M}_{p}\right)_{l k}=\frac{2 k-1+m+2 p+2-2 k-1}{p}=\frac{2 p+m}{p}=\frac{q}{p} .
$$

Now let us consider the case $q=2 p-m, m \in \llbracket 3, p-1 \rrbracket$ and suppose that $d>1$ : then $\min (m, m-2+d)=m$ and $\max (p-m+1, p-m+3-d)=p-m+1$. Then for all $k=1, p$

$$
\begin{aligned}
\sum_{l=1}^{q} M_{l k}= & \sum_{l=1}^{p-m+1}\left(M_{p-m+1}\right)_{l k}+\sum_{l=1}^{m-2}\left(M_{m-2}^{\prime \prime}\right)_{l k}+\sum_{l=1}^{p-m+1}\left(\widetilde{M}_{p-m+1}\right)_{l k} \\
& = \begin{cases}\frac{2(k-1)+2(p+1-k)-m}{p}=\frac{2 p-m}{p}=\frac{q}{p} & \text { if } k<m \\
\frac{2 k-m+m-2+2(p+1-k)-m}{p}=\frac{2 p-m}{p}=\frac{q}{p} & \text { if } m \leq k \leq p-m+1 . \\
\frac{2 k-m+2(p-k)}{p}=\frac{2 p-m}{p}=\frac{q}{p} & \text { if } k>p-m+1\end{cases}
\end{aligned}
$$

One reaches the same result in the specific cases $d \leq 1$ by noticing that for $l=1, p-m+1,\left(M_{p-m+1}\right)_{l k}=$ $\left(\widetilde{M}_{p-m+1}\right)_{l k}=0$ for $k=m-2+d, m-1$.

It follows that

Proposition 3. For all $p, q>0$ let $M \in \mathcal{M}_{q, p}(\mathbb{R})$ be a matrix which satisfies the properties of proposition 2. Then

$$
\left\|M^{\top} M\right\|_{2}=\max \left(\frac{q}{p}, \frac{p}{q}\right) .
$$

Proof. Define $A:=M^{\top} M$, it holds $\|A\|_{2}=\lambda_{\max }=\max _{\lambda \in \sigma(A)} \lambda(A)>0$, where $\sigma(A)$ is the set of eigenvalues of $A$. Using Gershgorin circle theorem, one bounds the spectrum of $A$ as follows:

$$
\forall \lambda \in \sigma(A), \forall k=1, p,\left|\lambda-A_{k k}\right| \leq \sum_{l \neq k} A_{k l},
$$

then

$$
\begin{aligned}
\lambda_{\max } & \leq \max _{k=1, p}\left(A_{k k}+\sum_{l \neq k} A_{k l}\right)=\max _{k=1, p}\left(\sum_{l=1, p} A_{k l}\right) \leq \max _{k=1, p}\left(\sum_{l=1}^{p} \sum_{h=1}^{q}\left(M^{\top}\right)_{k h} M_{h l}\right)=\max _{k=1, p}\left(\sum_{l=1}^{p} \sum_{h=1}^{q} M_{h k} M_{h l}\right) \\
& \leq \max _{k=1, p}\left(\sum_{h=1}^{q}\left(\sum_{l=1}^{p} M_{h l}\right) M_{h k}\right) .
\end{aligned}
$$


Due to the second and the third properties in proposition 2 satisfied by $M$, one finds

$$
\lambda_{\max } \leq \max _{k=1, p}\left(\max \left(1, \frac{p}{q}\right) \sum_{h=1}^{q} M_{h k}\right) \leq \max \left(1, \frac{p}{q}\right) \max \left(1, \frac{q}{p}\right)=\max \left(\frac{p}{q}, \frac{q}{p}\right) .
$$

Let us prove that $\lambda_{\max }=\max \left(\frac{p}{q}, \frac{q}{p}\right)$. Consider the vector $\vec{W} \in \mathbb{R}^{p}$ such that $\vec{W}=(1, \ldots, 1)^{\top}$. Suppose for instance that $p \leq q$, then using again the second and the third properties in proposition 2, we get for all $j=1, p$

$$
\begin{aligned}
\left(\left(M^{\top} M\right) \vec{W}\right)_{j} & =\sum_{k=1}^{p}\left(M^{\top} M\right)_{j k} W_{k}=\sum_{k=1}^{p} \sum_{h=1}^{q} M_{h k} M_{h j}=\sum_{h=1}^{q}\left(\sum_{k=1}^{p} M_{h k}\right) M_{h j} \\
& =\max \left(1, \frac{p}{q}\right)\left(\sum_{h=1}^{q} M_{h j}\right)=\max \left(\frac{p}{q}, \frac{q}{p}\right)=\max \left(\frac{p}{q}, \frac{q}{p}\right) W_{j}
\end{aligned}
$$

namely $\left(M^{\top} M\right) \vec{W}=\max \left(\frac{p}{q}, \frac{q}{p}\right) \vec{W}$.

Consequently, the proposed operator $\mathrm{R}$ in (33) is of optimal norm. One proceeds similarly for $\mathrm{R}^{\prime}$ by considering $M^{\top}$ instead of $M$ as the roles of $p$ and $q$ are exchanged:

$$
\|\mathrm{R}\|^{2}=\left\|M^{\top} M\right\|_{2}=\max \left(\frac{p}{q}, \frac{q}{p}\right), \quad \text { and } \quad\left\|\mathrm{R}^{\prime}\right\|^{2}=\left\|M M^{\top}\right\|_{2}=\max \left(\frac{p}{q}, \frac{q}{p}\right) .
$$

Remark 4. There is always a unit entry in $M$ according to proposition 2 (denoted by $M_{k_{0} l_{0}}$ ), one readily checks that if $w_{1}^{0} \in V_{1}$ with $\operatorname{supp}\left(w_{1}^{0}\right) \subset \Omega_{1}^{k_{0}}$, then it follows $\left\|\nabla\left(\mathrm{R} w_{1}^{0}\right)\right\|_{\Omega_{2}}^{2} \geq\left\|\nabla w_{1}^{0}\right\|_{\Omega_{1}}^{2}$. Hence $\|\mathrm{R}\|^{2} \geq 1$. Similarly, $\left\|\mathrm{R}^{\prime}\right\|^{2} \geq 1$.

\section{A.2 Weighted estimates for operators $R$}

In $\S$ A.1 we provided bounds for the norms of the geometry-based operators R. Here we provide bounds for the operator norm when we use a localization process (see section 4), that is when the operator R is locally applied in the neighborhood of the interface thanks to a cut-off function $\xi$ (defined as in section 4) whose support is localized either near a corner (proposition 4) or a straight line (proposition 5) of the interface $\Sigma$. We use the same notations as in $\S$ A.1.

Proposition 4. Let $\xi$ be a smooth positive function with support $S$, that depends only on the distance to the corner of angle $\alpha=2 \pi \frac{p}{p+q}$. Then

$$
\forall w_{1} \in V_{1}, \quad\left\|\xi^{1 / 2} \nabla\left(\mathrm{R} w_{1}\right)\right\|_{\Omega_{2} \cap S}^{2} \leq I_{\alpha}\left\|\xi^{1 / 2} \nabla w_{1}\right\|_{\Omega_{1} \cap S}^{2},
$$

where $I_{\alpha}=\max \left(\frac{p}{q}, \frac{q}{p}\right)$, and $\mathrm{R}$ is a rotation- and symmetry-based operator from $V_{1}$ to $V_{2}$ defined as in (21).

Proof. Let $w_{1} \in V_{1}$.

$$
\begin{aligned}
\left\|\xi^{1 / 2} \nabla\left(\mathrm{R} w_{1}\right)\right\|_{\Omega_{2} \cap S}^{2} & =\int_{\Omega_{2} \cap S} \xi(\rho)\left(\left|\frac{\partial\left(\mathrm{R} w_{1}\right)}{\partial \rho}\right|^{2}+\frac{1}{\rho^{2}}\left|\frac{\partial\left(\mathrm{R} w_{1}\right)}{\partial \theta}\right|^{2}\right) \rho d \rho d \theta \\
& =\sum_{l=1}^{q} \int_{\Omega_{2}^{l} \cap S} \xi(\rho)\left(\left|\frac{\partial\left(\mathrm{R} w_{1}\right)}{\partial \rho}\right|^{2}+\frac{1}{\rho^{2}}\left|\frac{\partial\left(\mathrm{R} w_{1}\right)}{\partial \theta}\right|^{2}\right) \rho d \rho d \theta .
\end{aligned}
$$

Then similar to (34) with the change of variables $(\rho, \theta) \mapsto\left(r_{k}, \varphi_{k}\right)$ for $k=1, p$ that maps $\Omega_{2}^{l}$ to $\Omega_{1}^{k}$ (note that 
$\left.\xi\left(r_{k}\right)=\xi(\rho)\right)$ one finds

$$
\begin{aligned}
\left\|\xi^{1 / 2} \nabla\left(\mathrm{R} w_{1}\right)\right\|_{\Omega_{2} \cap S}^{2} & \leq \sum_{l=1}^{q}\left(\sum_{k=1}^{p} M_{l k}\left(\int_{\Omega_{1}^{k} \cap S} \xi\left(r_{k}\right)\left(\left|\frac{\partial w_{1}^{k}\left(r_{k}, \varphi_{k}\right)}{\partial r_{k}}\right|^{2}+\frac{1}{r_{k}^{2}}\left|\frac{\partial w_{1}^{k}\left(r_{k}, \varphi_{k}\right)}{\partial \varphi_{k}}\right|^{2}\right) r_{k} d r_{k} d \varphi_{k}\right)^{1 / 2}\right)^{2} \\
& \leq \sum_{l=1}^{q}\left(\sum_{k=1}^{p} M_{l k}\left\|\xi^{1 / 2} \nabla w_{1}^{k}\right\|_{\Omega_{1}^{k} \cap S}\right)^{2}
\end{aligned}
$$

Introducing $\vec{W}_{\xi}=\left(\left\|\xi^{1 / 2} \nabla w_{1}^{1}\right\|_{\Omega_{1}^{1} \cap S}, \ldots,\left\|\xi^{1 / 2} \nabla w_{1}^{p}\right\|_{\Omega_{1}^{p} \cap S}\right)^{\top}$, then one has $M \vec{W}_{\xi}=\sum_{k=1}^{p} M_{l k}\left\|\xi^{1 / 2} \nabla w_{1}^{k}\right\|_{\Omega_{1}^{k} \cap S}$, using proposition 3 one finally gets

$$
\left\|\xi^{1 / 2} \nabla\left(\mathrm{R} w_{1}\right)\right\|_{\Omega_{2} \cap S}^{2} \leq\left(M^{\top} M \vec{W}_{\xi}, \vec{W}_{\xi}\right) \leq I_{\alpha}\left\|\xi^{1 / 2} \nabla w_{1}\right\|_{\Omega_{1} \cap S}^{2} .
$$

Remark 5. Following remark 4, one can find $w_{1}^{0} \in V_{1}$ such that $\left\|\xi^{1 / 2} \nabla\left(\mathrm{R} w_{1}^{0}\right)\right\|_{\Omega_{2} \cap S}^{2} \geq\left\|\xi^{1 / 2} \nabla w_{1}^{0}\right\|_{\Omega_{1} \cap S}^{2}$.

Proposition 5. Let $\xi$ be a smooth positive function with support $S$ that is symmetric with respect to the interface. Then for all $w_{1} \in V_{1}$

$$
\left\|\xi^{1 / 2} \nabla\left(\mathrm{R} w_{1}\right)\right\|_{\Omega_{2} \cap S}^{2}=\left\|\xi^{1 / 2} \nabla w_{1}\right\|_{\Omega_{1} \cap S}^{2},
$$

where $\mathrm{R}$ is the symmetry-based operator (22).

Proof. This inequality is obtained using the change of variables $\left(x^{\Sigma}, y^{\Sigma}\right) \rightarrow\left(x^{\Sigma},-y^{\Sigma}\right)$ in $\Omega_{2} \cap S$. For all $w_{1} \in V_{1}$

$$
\begin{aligned}
\left\|\xi^{1 / 2} \nabla\left(\mathrm{R} w_{1}\right)\right\|_{\Omega_{2} \cap S}^{2} & =\int_{\Omega_{2} \cap S} \xi\left(x^{\Sigma}, y^{\Sigma}\right)\left(\left|\frac{\partial\left(\mathrm{R} w_{1}\right)}{\partial x^{\Sigma}}\right|^{2}+\left|\frac{\partial\left(\mathrm{R} w_{1}\right)}{\partial y^{\Sigma}}\right|^{2}\right) d x^{\Sigma} d y^{\Sigma} \\
& =\int_{\Omega_{1} \cap S} \xi\left(x^{\Sigma}, y^{\Sigma}\right)\left(\left|\frac{\partial w_{1}}{\partial x^{\Sigma}}\right|^{2}+\left|\frac{\partial w_{1}}{\partial y^{\Sigma}}\right|^{2}\right) d x^{\Sigma} d y^{\Sigma}=\left\|\xi^{1 / 2} \nabla w_{1}\right\|_{\Omega_{1} \cap S}^{2}
\end{aligned}
$$

\section{A.3 Local and global interpolation estimates}

Let $\hat{\tau}$ be the reference triangle, with corners $(1,0),(0,1)$ and $(0,0)$, and let $\hat{I}_{d}$ be the Lagrange interpolation operator over $\mathbb{P}_{d}(\hat{\tau})$. Given $\left(\mathcal{T}_{h}\right)_{h}$ a regular family of triangulations of a domain $\Omega$ we call $I_{\tau}^{d}$ the Lagrange interpolation operator over $\mathbb{P}_{d}(\tau)$, for all $h$ and all $\tau \in \mathcal{T}_{h}$.

Lemma 4. On the reference triangle $\hat{\tau}$, one has the estimate

$$
\exists \hat{C}>0, \forall \hat{\chi} \in \mathbb{P}_{1}(\hat{\tau}), \forall \hat{v} \in \mathbb{P}_{d}(\hat{\tau}),\left\|\hat{\nabla}\left(\hat{\chi} \hat{v}-\hat{I}_{d}(\hat{\chi} \hat{v})\right)\right\|_{\hat{\tau}} \leq \hat{C}|\hat{\chi}|_{W^{1, \infty}(\hat{\tau})}\|\hat{\nabla} \hat{v}\|_{\hat{\tau}} .
$$

As a consequence, one has the uniform local estimate

$$
\exists C>0, \forall h, \forall \tau \in \mathcal{T}_{h}, \forall \chi \in \mathbb{P}_{1}(\tau), \forall v \in \mathbb{P}_{d}(\tau),\left\|\nabla\left(\chi v-I_{\tau}^{d}(\chi v)\right)\right\|_{\tau} \leq C h_{\tau}|\chi|_{W^{1, \infty}(\tau)}\|\nabla v\|_{\tau} .
$$

Proof. Write $\hat{\chi}(\hat{x}, \hat{y})=\alpha+\hat{\chi}_{1}(\hat{x}, \hat{y})$, resp. $\hat{v}(\hat{x}, \hat{y})=\hat{v}_{-}(\hat{x}, \hat{y})+\hat{v}_{d}(\hat{x}, \hat{y})$ where $\hat{\chi}_{1}(\hat{x}, \hat{y})=\beta \hat{x}+\gamma \hat{y}$, resp. $\hat{v}_{d}(\hat{x}, \hat{y})=\sum_{i=0, d} a_{i} \hat{x}^{i} \hat{y}^{d-i}$ and $\operatorname{deg}\left(\hat{v}_{-}\right)<d$. For $i=0, d$, define $\hat{e}_{i}(\hat{x}, \hat{y})=\hat{x}^{i+1} \hat{y}^{d-i}-\hat{I}_{d}\left(\hat{x}^{i+1} \hat{y}^{d-i}\right)$, resp. $\hat{f}_{i}(\hat{x}, \hat{y})=\hat{x}^{i} \hat{y}^{d+1-i}-\hat{I}_{d}\left(\hat{x}^{i} \hat{y}^{d+1-i}\right)$. Note that $|\hat{\chi}|_{W^{1, \infty}(\hat{\tau})}=\max (|\beta|,|\gamma|)$. 
Since there holds $\hat{\chi} \hat{v}-\hat{I}_{d}(\hat{\chi} \hat{v})=\hat{\chi}_{1} \hat{v}_{d}-\hat{I}_{d}\left(\hat{\chi}_{1} \hat{v}_{d}\right)=\beta \sum_{i=0, d} a_{i} \hat{e}_{i}(\hat{x}, \hat{y})+\gamma \sum_{i=0, d} a_{i} \hat{f}_{i}(\hat{x}, \hat{y})$, one finds

$$
\begin{aligned}
\left|\hat{\nabla}\left(\hat{\chi} \hat{v}-\hat{I}_{d}(\hat{\chi} \hat{v})\right)(\hat{x}, \hat{y})\right| & \leq|\beta| \sum_{i=0, d}\left|a_{i}\right|\left|\hat{\nabla} \hat{e}_{i}(\hat{x}, \hat{y})\right|+|\gamma| \sum_{i=0, d}\left|a_{i}\right|\left|\hat{\nabla} \hat{f}_{i}(\hat{x}, \hat{y})\right| \\
& \leq|\hat{\chi}|_{W^{1, \infty}(\hat{\tau})} \sum_{i=0, d}\left|a_{i}\right|\left(\left|\hat{\nabla} \hat{e}_{i}(\hat{x}, \hat{y})\right|+\left|\hat{\nabla} \hat{f}_{i}(\hat{x}, \hat{y})\right|\right) \\
& \leq|\hat{\chi}|_{W^{1, \infty}(\hat{\tau})}\left(\sum_{i=0, d}\left|a_{i}\right|^{2}\right)^{1 / 2}\left(\sum_{i=0, d}\left(\left|\hat{\nabla} \hat{e}_{i}(\hat{x}, \hat{y})\right|+\left|\hat{\nabla} \hat{f}_{i}(\hat{x}, \hat{y})\right|\right)^{2}\right)^{1 / 2} .
\end{aligned}
$$

It follows that $\left\|\hat{\nabla}\left(\hat{\chi} \hat{v}-\hat{I}_{d}(\hat{\chi} \hat{v})\right)\right\|_{\hat{\tau}} \leq \hat{C}_{d}|\hat{\chi}|_{W^{1, \infty}(\hat{\tau})}\left(\sum_{i=0, d}\left|a_{i}\right|^{2}\right)^{1 / 2}$, with $\hat{C}_{d}=\sqrt{2}\left[\sum_{i=0, d}\left(\left\|\hat{\nabla} \hat{e}_{i}\right\|_{\hat{\tau}}^{2}+\left\|\hat{\nabla} \hat{f}_{i}\right\|_{\hat{\tau}}^{2}\right)\right]^{1 / 2}$. Then, we remark that the $\ell^{2}$-norm of the coefficients is a norm over $\mathbb{P}_{d}(\hat{\tau})$, hence it is also a norm over its vector subspace $\mathbb{P}_{d}^{z m v}(\hat{\tau})$ made of zero-mean value polynomials on $\hat{\tau}$. Now, over $\mathbb{P}_{d}^{z m v}(\hat{\tau})$, the semi-norm $\|\hat{\nabla} \cdot\|_{\hat{\tau}}$ is also a norm and because $\mathbb{P}_{d}^{z m v}(\hat{\tau})$ is a finite dimensional vector space, both norms are equivalent. Noting finally that, starting from $\hat{v}$, one has only to modify the degree- 0 coefficient to obtain a zero-mean value polynomial, we finally get that there exists $\hat{C}^{\prime}$ independent of $\hat{v}$ such that $\left(\sum_{i=0, d}\left|a_{i}\right|^{2}\right)^{1 / 2} \leq \hat{C^{\prime}}\|\hat{\nabla} \hat{v}\|_{\hat{\tau}}$. Taking $\hat{C}=\hat{C}_{d} \hat{C}^{\prime}$ leads to (35).

We recall that provided the family of triangulations $\left(\mathcal{T}_{h}\right)_{h}$ is regular, there exists $\mathbf{s}>0$ such that, for all $h$ and for all $\tau \in \mathcal{T}_{h}$, there holds $h_{\tau} \leq \mathbf{s} \rho_{\tau}$, where $\rho_{\tau}$ is the diameter of the largest ball that can be inscribed in $\tau$. One can then derive (36) from (35) by using the affine mapping from the reference triangle $\hat{\tau}$ to the triangle $\tau \in \mathcal{T}_{h}$. We refer for instance to [16]. We report here the computations for the sake of completeness $\left(C_{0}, C_{1}, \cdots\right.$ are constants that are independent of $h$ and $\left.\tau\right)$ :

$$
\begin{aligned}
\left\|\nabla\left(\chi v-I_{\tau}^{d}(\chi v)\right)\right\|_{\tau} & \leq C_{0} \mathrm{~s}\left\|\hat{\nabla}\left(\hat{\chi} \hat{v}-\hat{I}_{d}(\hat{\chi} \hat{v})\right)\right\|_{\hat{\tau}} & & \text { cf. Lemmas 1.100-1.101 of [16] } \\
& \leq C_{1}|\hat{\chi}|_{W^{1, \infty}(\hat{\tau})}\|\hat{\nabla} \hat{v}\|_{\hat{\tau}} & & \text { cf. (35) } \\
& \leq C_{2} h_{\tau}|\chi|_{W^{1, \infty}(\tau)} \times C_{3}\|\nabla v\|_{\tau} & & \text { cf. Lemmas 1.100-1.101 of [16], }
\end{aligned}
$$

that is (36) with $C=C_{2} C_{3}$.

Let us prove the estimate over $\Omega_{2}^{h}=\operatorname{int}\left(\bigcup_{p=1, P} \cup_{\tau \in \mathcal{T}_{h, 2}^{p}} \tau\right)$. Recall that $V:=H_{0}^{1}(\Omega), V_{(d)}^{h}:=\left\{v \in V:\left.v\right|_{\tau} \in\right.$ $\left.\mathbb{P}_{d}(\tau), \forall \tau \in \mathcal{T}_{h}\right\}$, and $I_{h}$ is the interpolation operator on $V_{(d)}^{h}$.

Lemma 5. Consider a cut-off function $\chi$, and denote by $\chi_{h}$ its interpolation of degree 1. For all $v^{h} \in V_{(d)}^{h}$,

$$
\exists C>0, \quad\left\|\nabla\left(\chi_{h} v^{h}-I_{h}\left(\chi_{h} v^{h}\right)\right)\right\|_{\Omega_{2}^{h}} \leq C h\|\chi\|_{W^{1, \infty}\left(\Omega_{2}\right)}\left\|\nabla v^{h}\right\|_{\Omega_{2}} .
$$

Proof. To obtain (37), we evaluate the $L^{2}$-norm of $\nabla\left(\chi_{h} v^{h}-I_{h}\left(\chi_{h} v^{h}\right)\right)$ on $\Omega_{2}^{h}$ by splitting $\Omega_{2}^{h}$ into triangles, and then going back to the reference triangle to use the uniform estimate (36):

$$
\left\|\nabla\left(\chi_{h} v^{h}-I_{h}\left(\chi_{h} v^{h}\right)\right)\right\|_{\Omega_{2}^{h}}^{2}=\sum_{\tau \subset \Omega_{2}^{h}} \int_{\tau}\left|\nabla\left(\chi_{h \mid \tau} v^{h}{ }_{\mid \tau}-I_{\tau}^{d}\left(\chi_{h \mid \tau} v^{h}{ }_{\mid \tau}\right)\right)\right|^{2} \leq C \sum_{\tau \subset \Omega_{2}^{h}} h_{\tau}^{2}\left|\chi_{h \mid \tau}\right|_{W^{1, \infty}(\tau)}^{2}\left\|\nabla v^{h}{ }_{\mid \tau}\right\|_{\tau}^{2} .
$$

Using the definition of the meshsize $h$ yields

$$
\left\|\nabla\left(\chi_{h} v^{h}-I_{h}\left(\chi_{h} v^{h}\right)\right)\right\|_{\Omega_{2}^{h}} \leq C h\left\|\chi_{h}\right\|_{W^{1, \infty}\left(\Omega_{2}\right)}\left\|\nabla v^{h}\right\|_{\Omega_{2}} .
$$

One concludes using the stability estimate (25). 